\title{
In situ observation of short- and long-timescale material property evolution under extreme conditions
}

\author{
Cody Andrew Dennett
}

November 2020

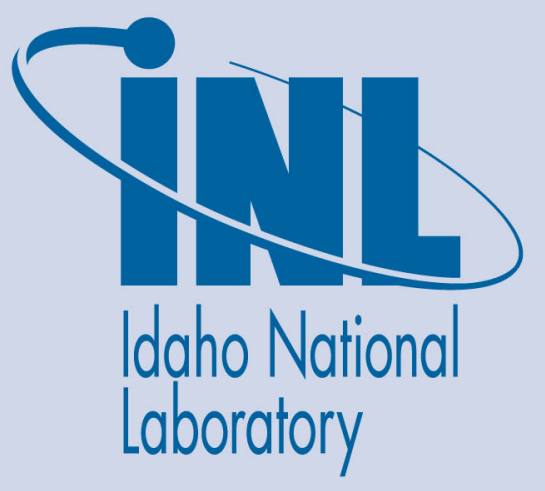

The INL is a U.S. Department of Energy National Laboratory operated by Battelle Energy Alliance 


\title{
In situ observation of short- and long-timescale material property evolution under extreme conditions
}

\author{
Cody Andrew Dennett
}

November 2020

Idaho National Laboratory Idaho Falls, Idaho 83415

http://www.inl.gov

Prepared for the U.S. Department of Energy Office of Nuclear Energy Under DOE Idaho Operations Office

Contract DE-AC07-05ID14517 


\section{In situ observation of short- and long- timescale material property evolution under extreme conditions}

\section{MS\&T 2020 - November, 2020}

\section{Cody A. Dennett}

Materials Science and Engineering Department Idaho National Laboratory

cody.dennett@inl.gov

This work was performed, in part, at the Center for

Integrated Nanotechnologies, an Office of Science User

Facility operated for the U.S. Department of Energy

(DOE) Office of Science. Sandia National Laboratories is a

multi-mission laboratory managed and operated by

National Technology and Engineering Solutions of

Sandia, LLC., a wholly owned subsidiary of Honeywell

International, Inc., for the U.S. DOE's National Nuclear

Security Administration under contract DE-NA-0003525.

The views expressed in the presentation do not

necessarly represent the views of the U.S. DOE or the 


\section{material performance is challenged under extreme environments}

temperature

corrosives

radiation

pressure

fatigue 
material performance is challenged under extreme environments

\section{temperature}

corrosives

radiation

pressure

fatigue 


\section{micro-scale}

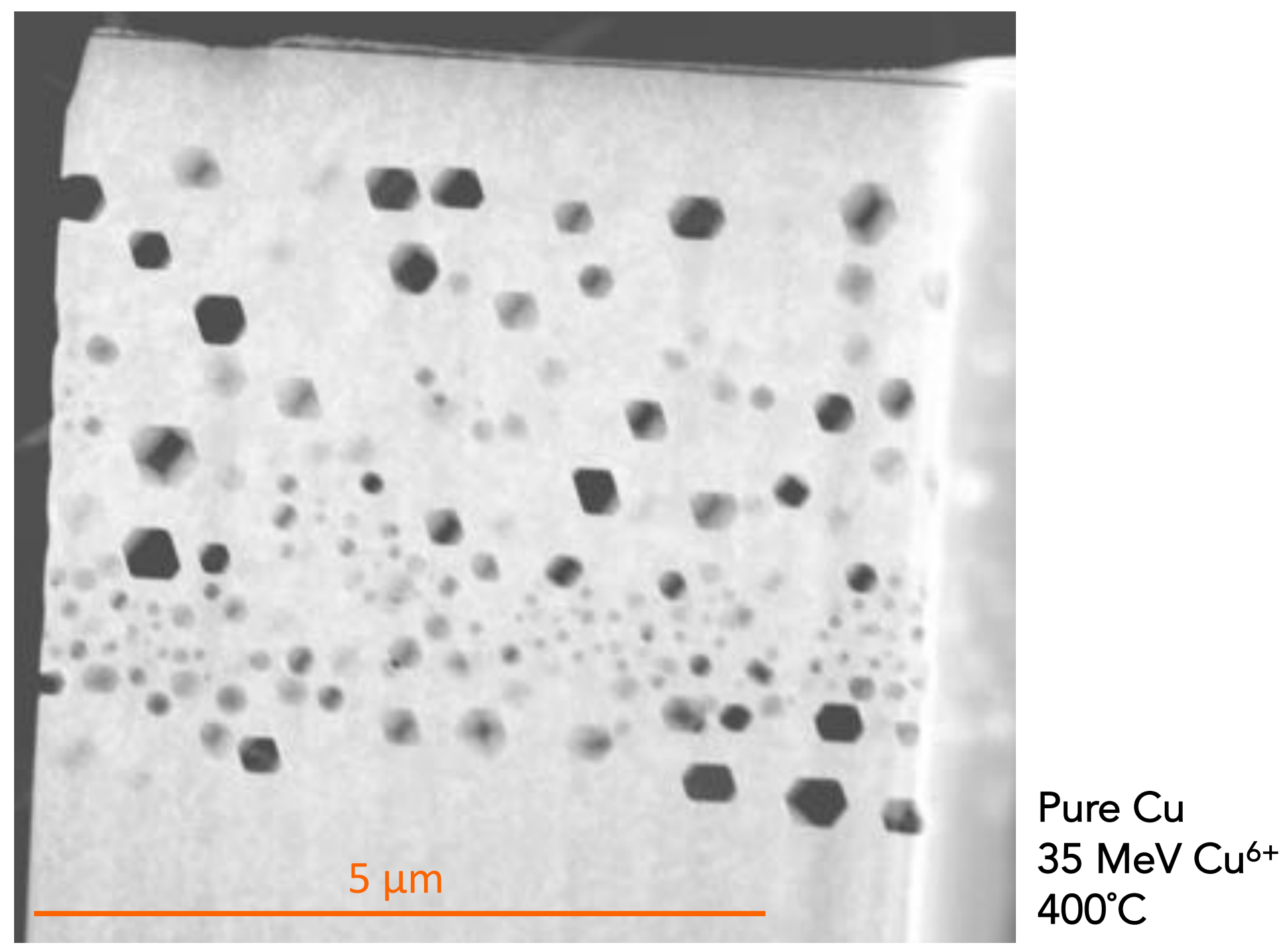




\section{micro-scale}
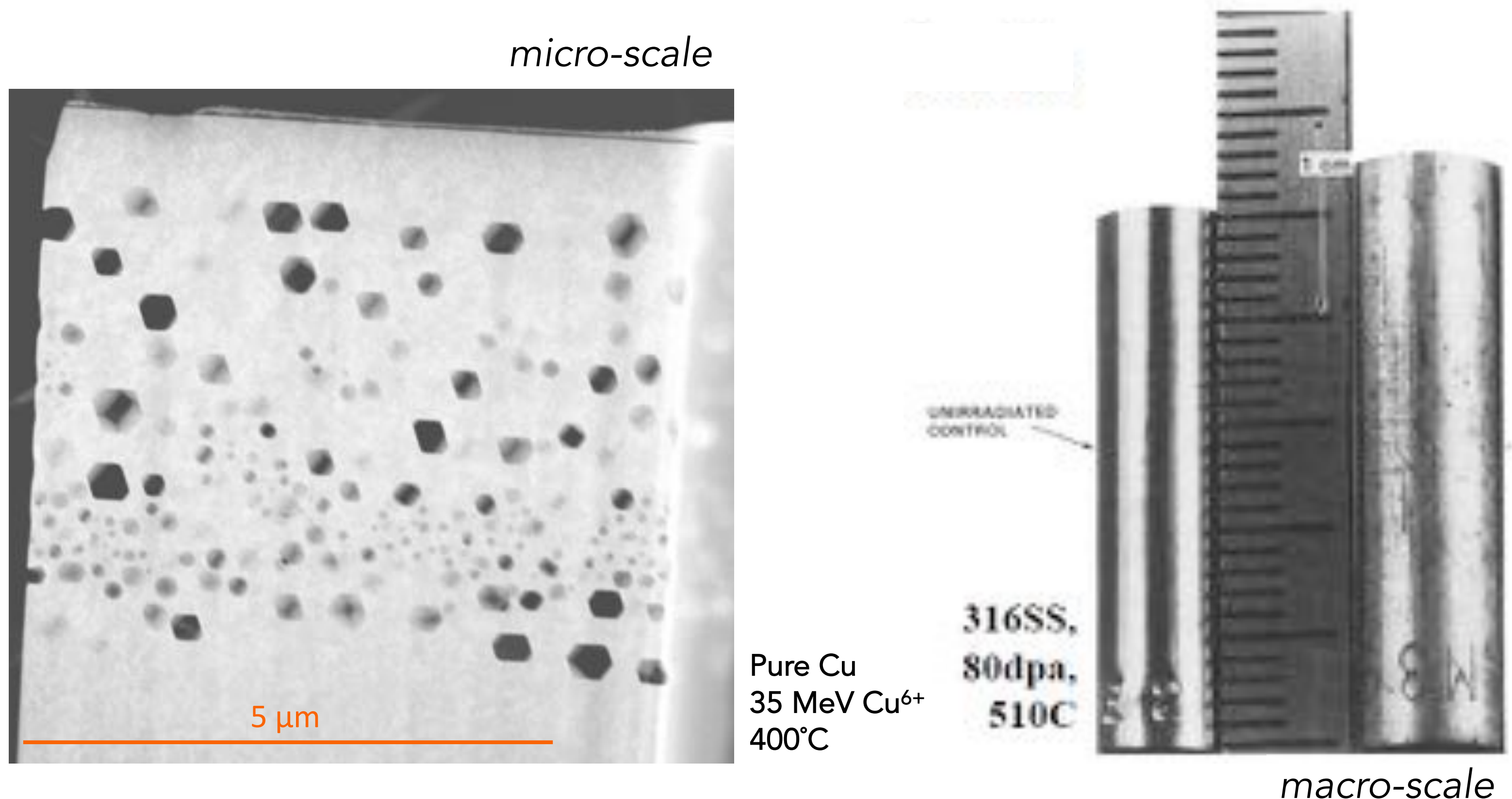

Pure $\mathrm{Cu}$ $35 \mathrm{MeV} \mathrm{Cu}^{6+}$ $400^{\circ} \mathrm{C}$ 


\title{
in situ characterization
}

\author{
material structure
}

material properties 


\title{
in situ characterization
}

\author{
material structure
}

material properties 
in situ TEM

Sandia Ion Beam Lab in situ Raman spectroscopy JANUUS-Saclay
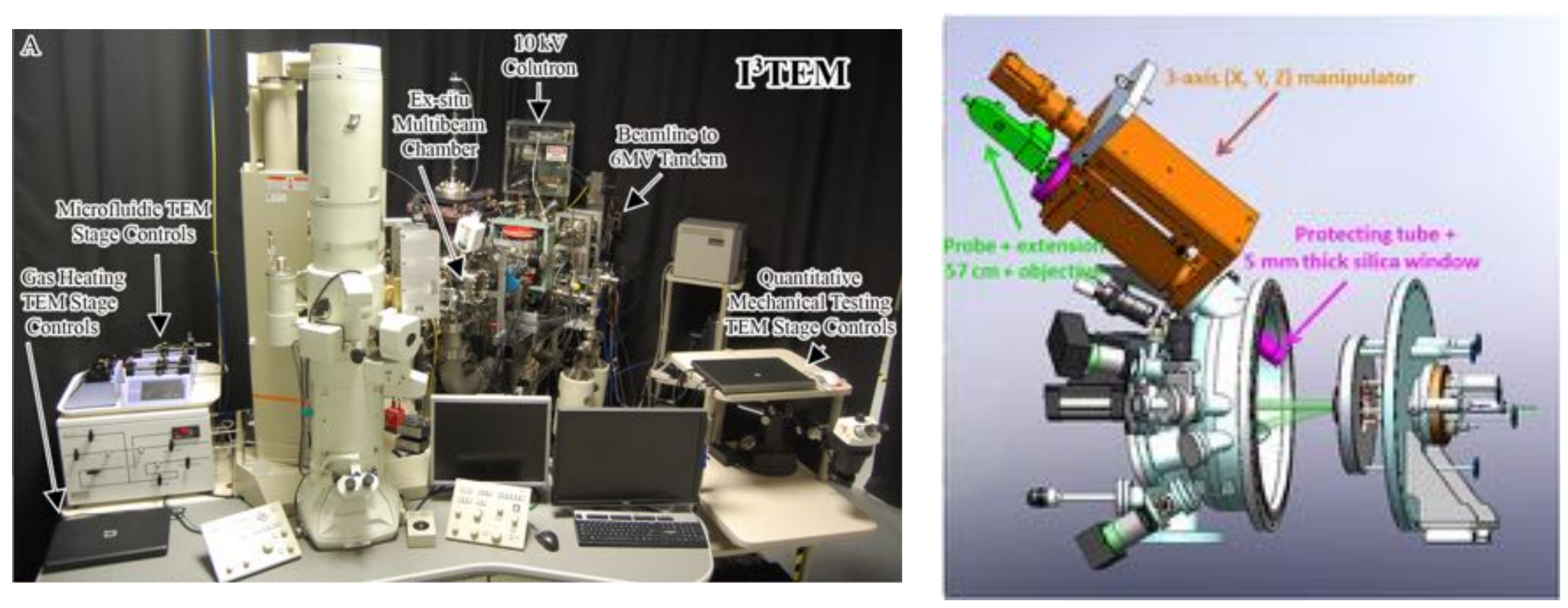


\title{
in situ characterization
}

\section{material structure}

\author{
material properties
}




\section{Transient Grating Spectroscopy (TGS)}

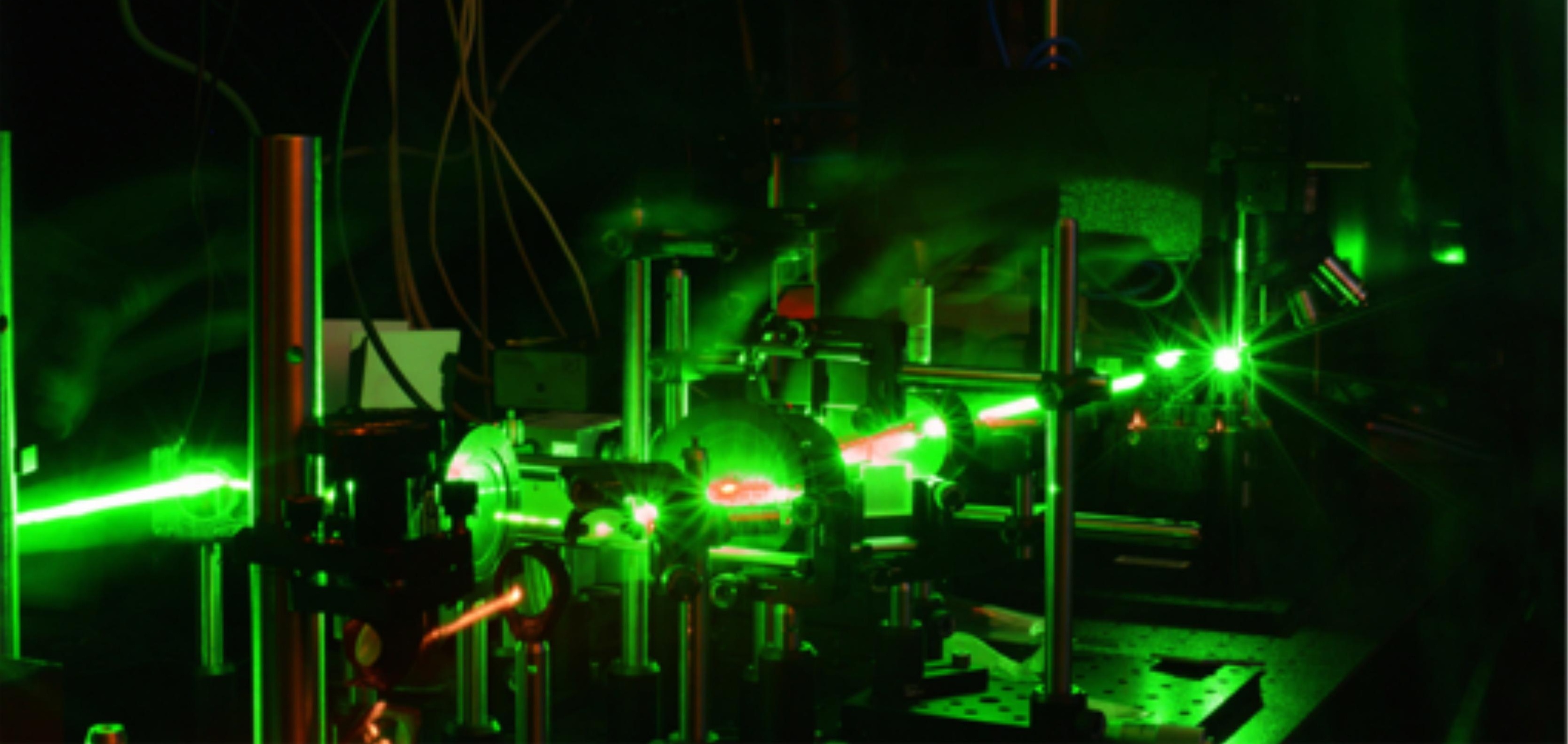




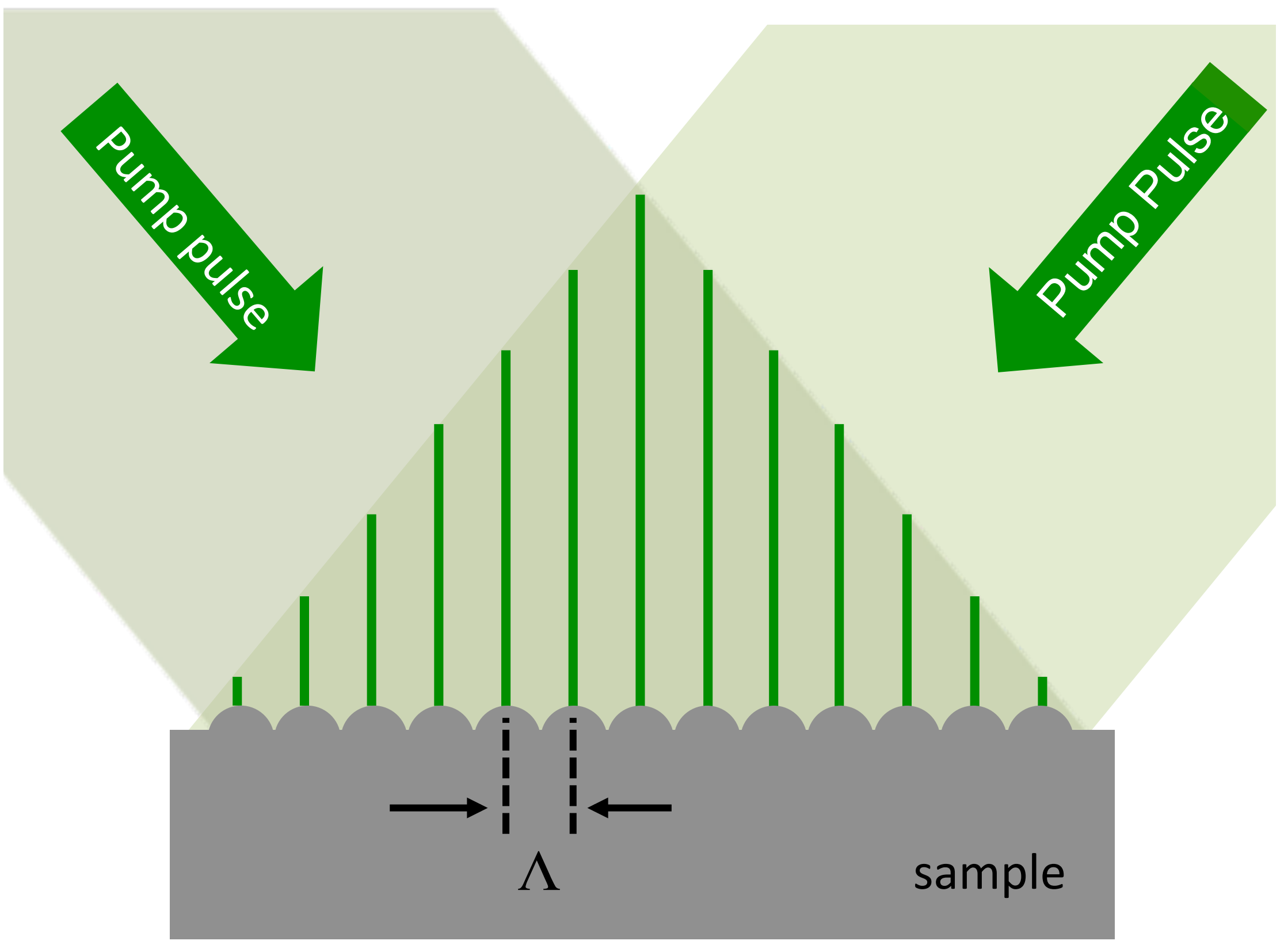




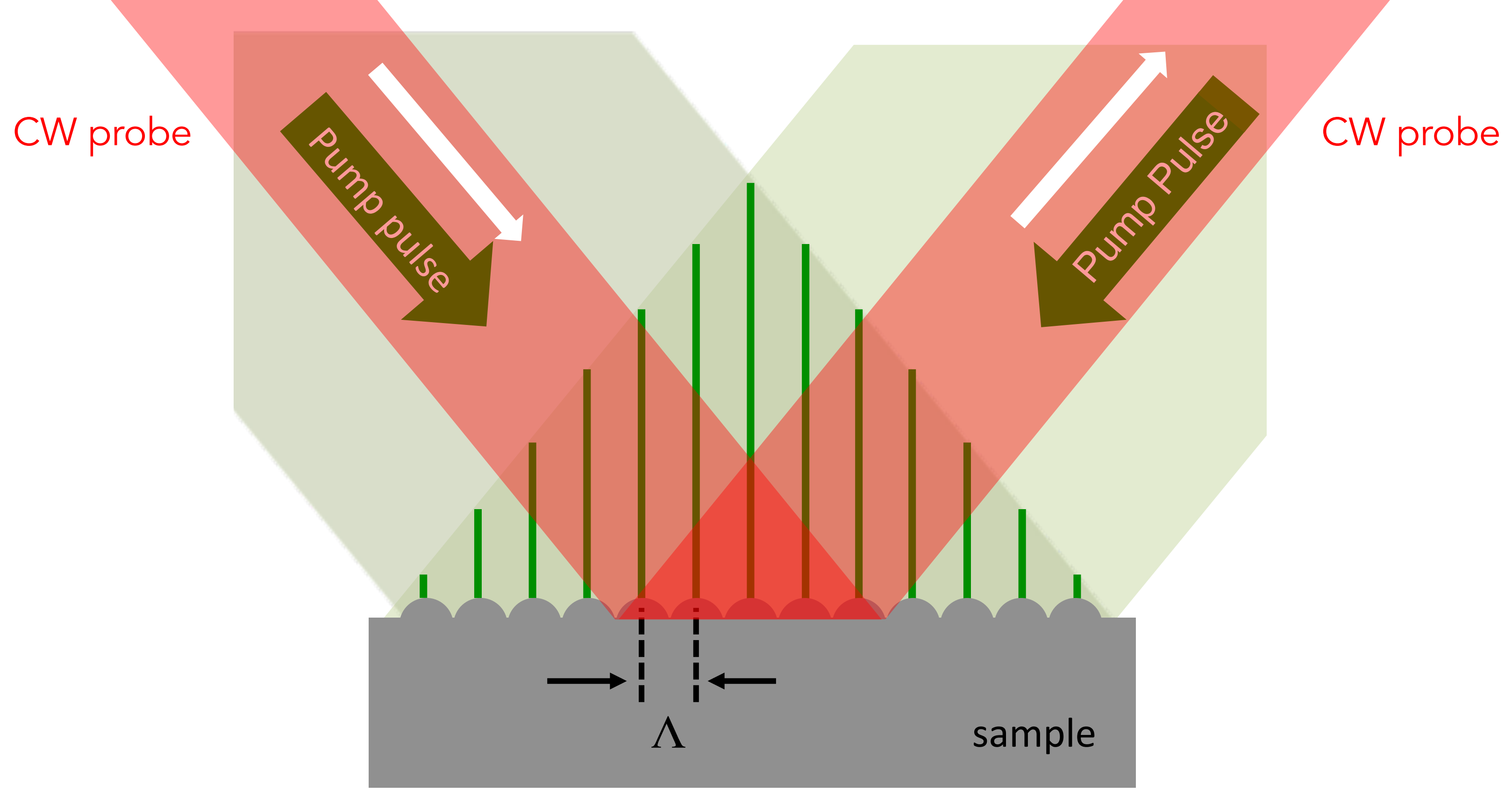




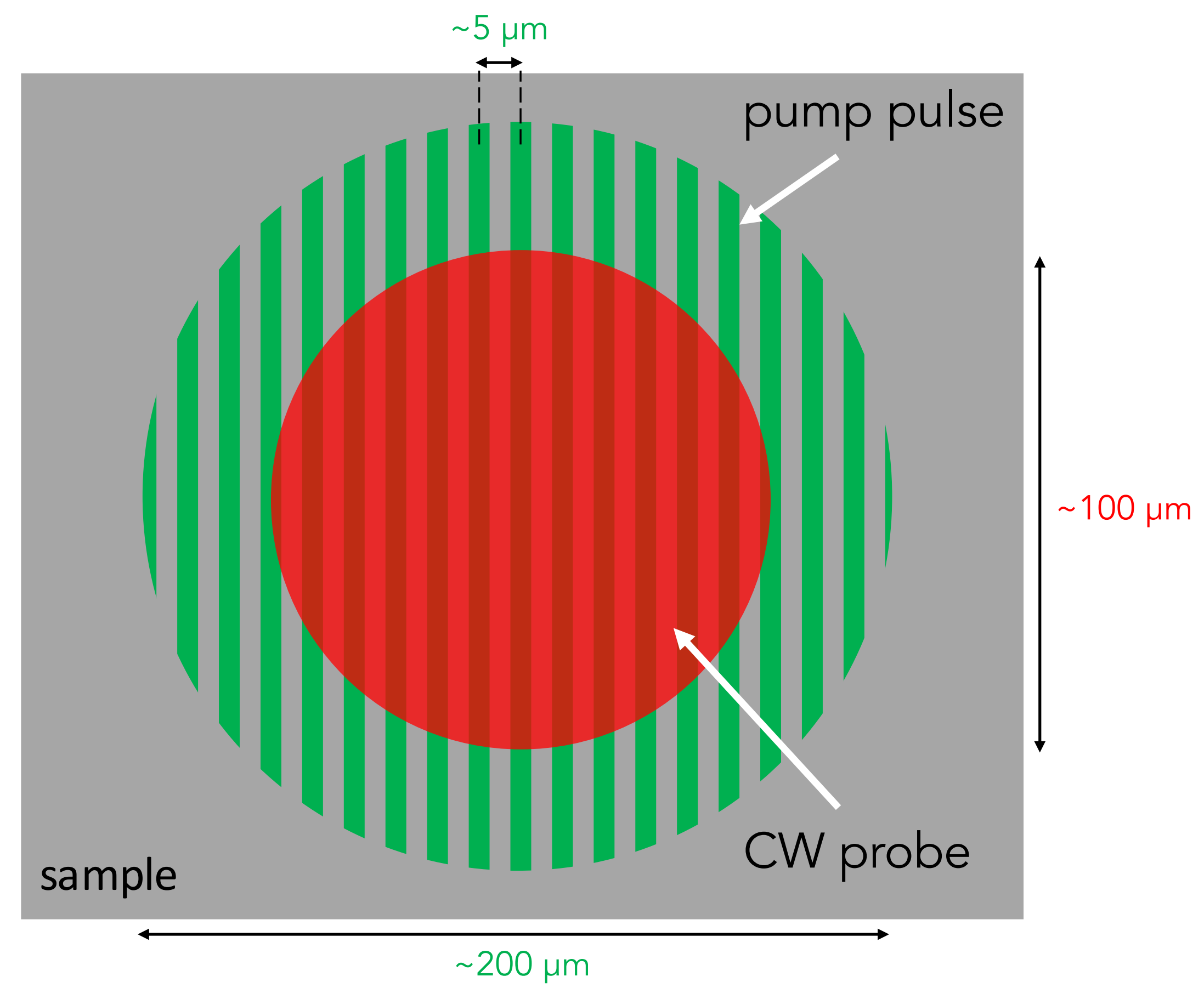




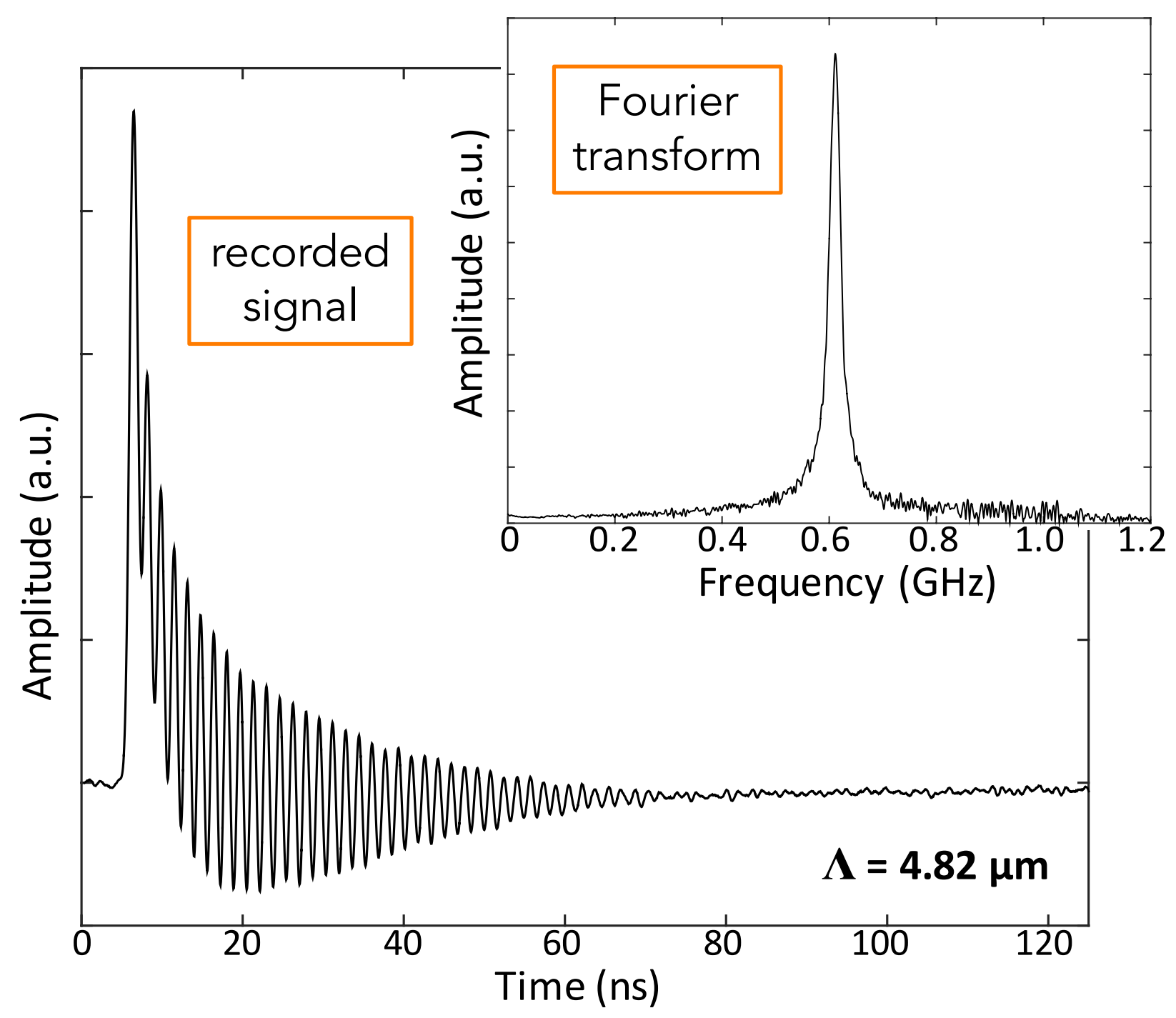




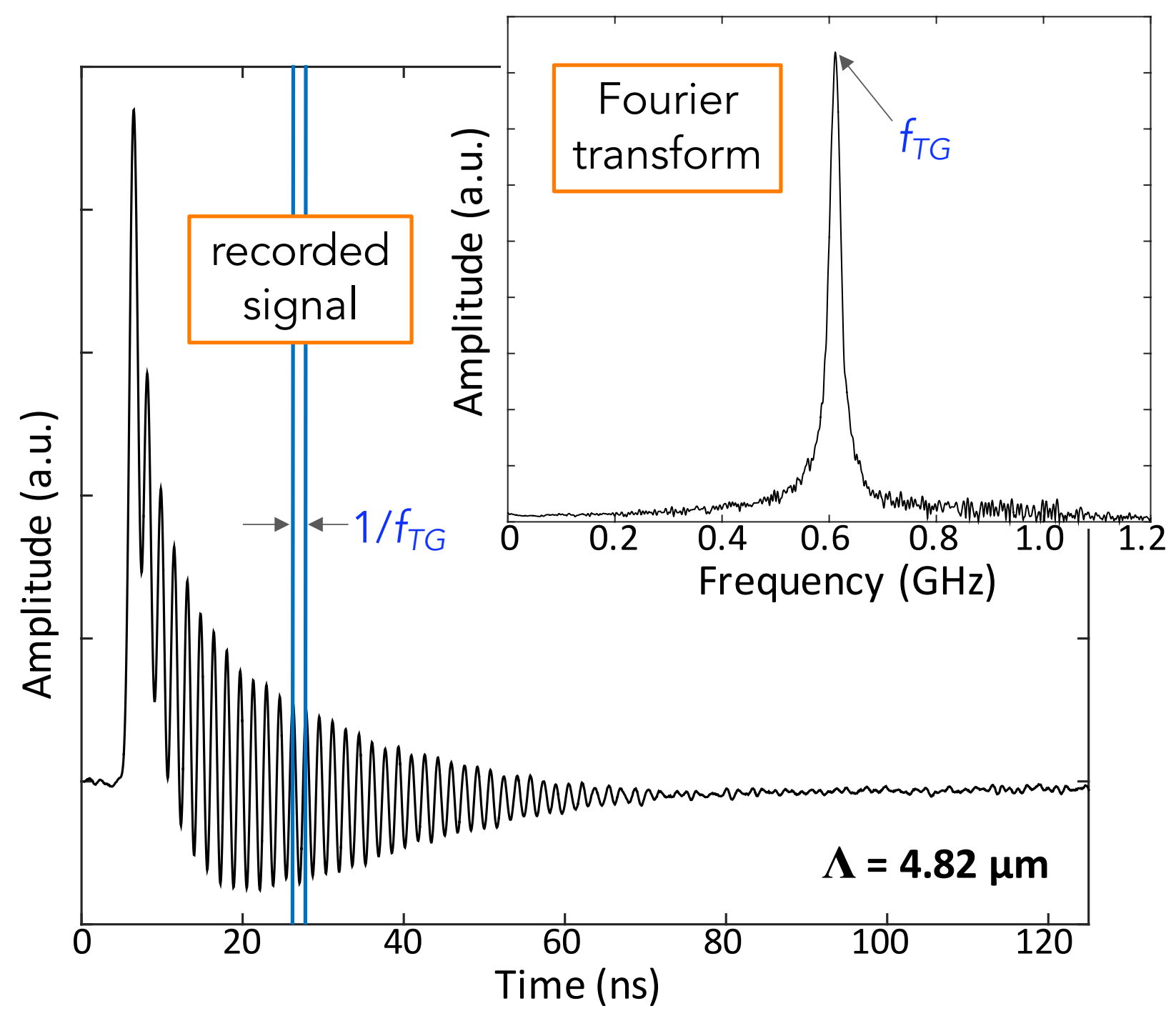

acoustic oscillations return elastic mechanical properties 


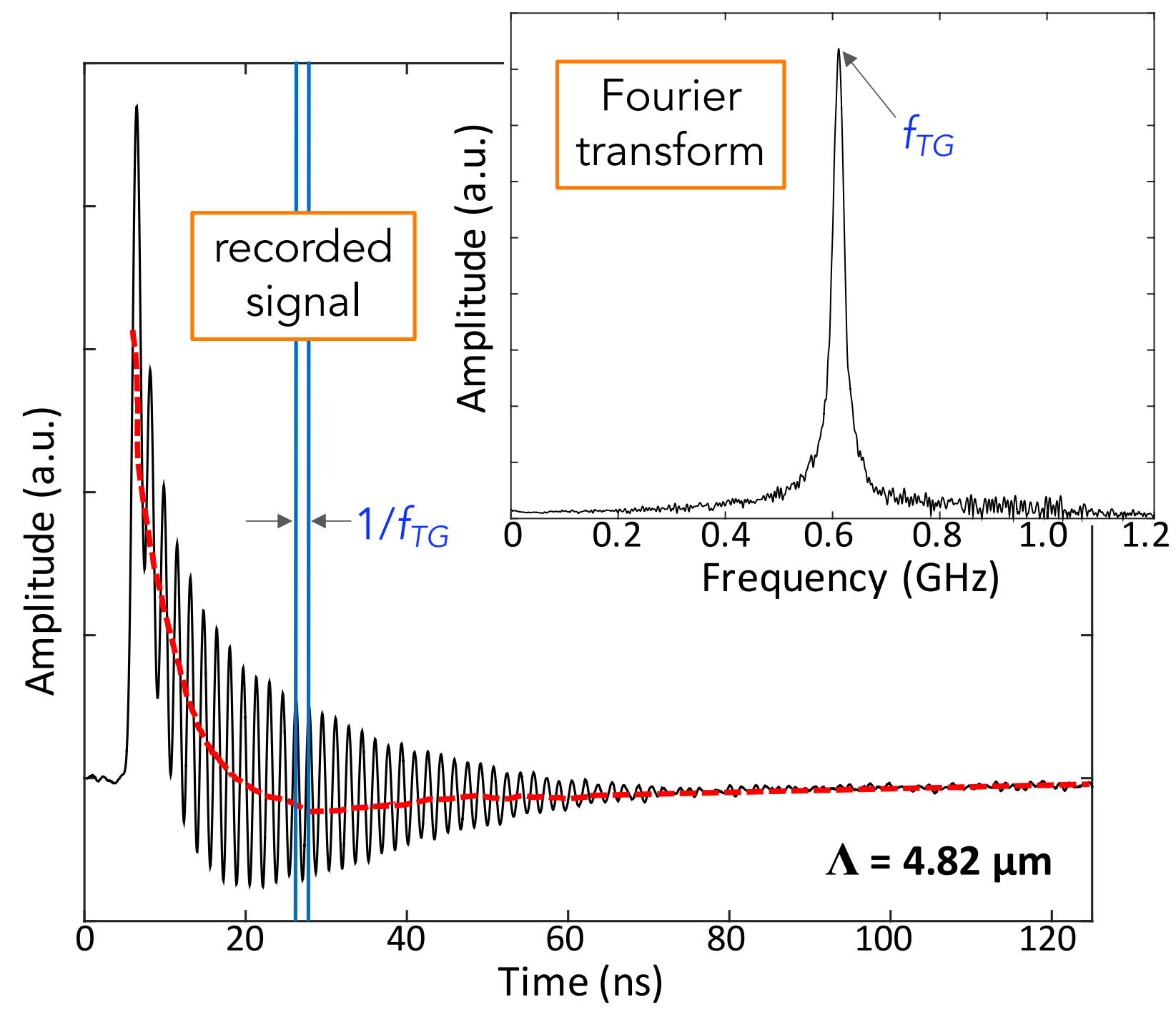

acoustic oscillations return elastic mechanical properties

grating decay returns thermal transport properties 
$35 \mathrm{MeV} \mathrm{Cu}^{6+}$ in $\mathrm{Cu}$ at $400^{\circ} \mathrm{C}$

\begin{tabular}{|c|c|}
\hline material & $\begin{array}{c}\text { single crystal } \\
\{111\} \mathrm{Cu}\end{array}$ \\
\hline ion species & $\mathrm{Cu}^{6+}$ \\
\hline ion energy & $35 \mathrm{MeV}$ \\
\hline temperature & $400^{\circ} \mathrm{C}$ \\
\hline spot size & $0.19 \mathrm{~cm}^{2}$ \\
\hline $\begin{array}{c}\text { beam current } \\
\text { target doses } \\
\text { (peak) }\end{array}$ & $\begin{array}{l}0,5,10,30 \mathrm{nA} \\
50,100 \mathrm{dpa}\end{array}$ \\
\hline
\end{tabular}

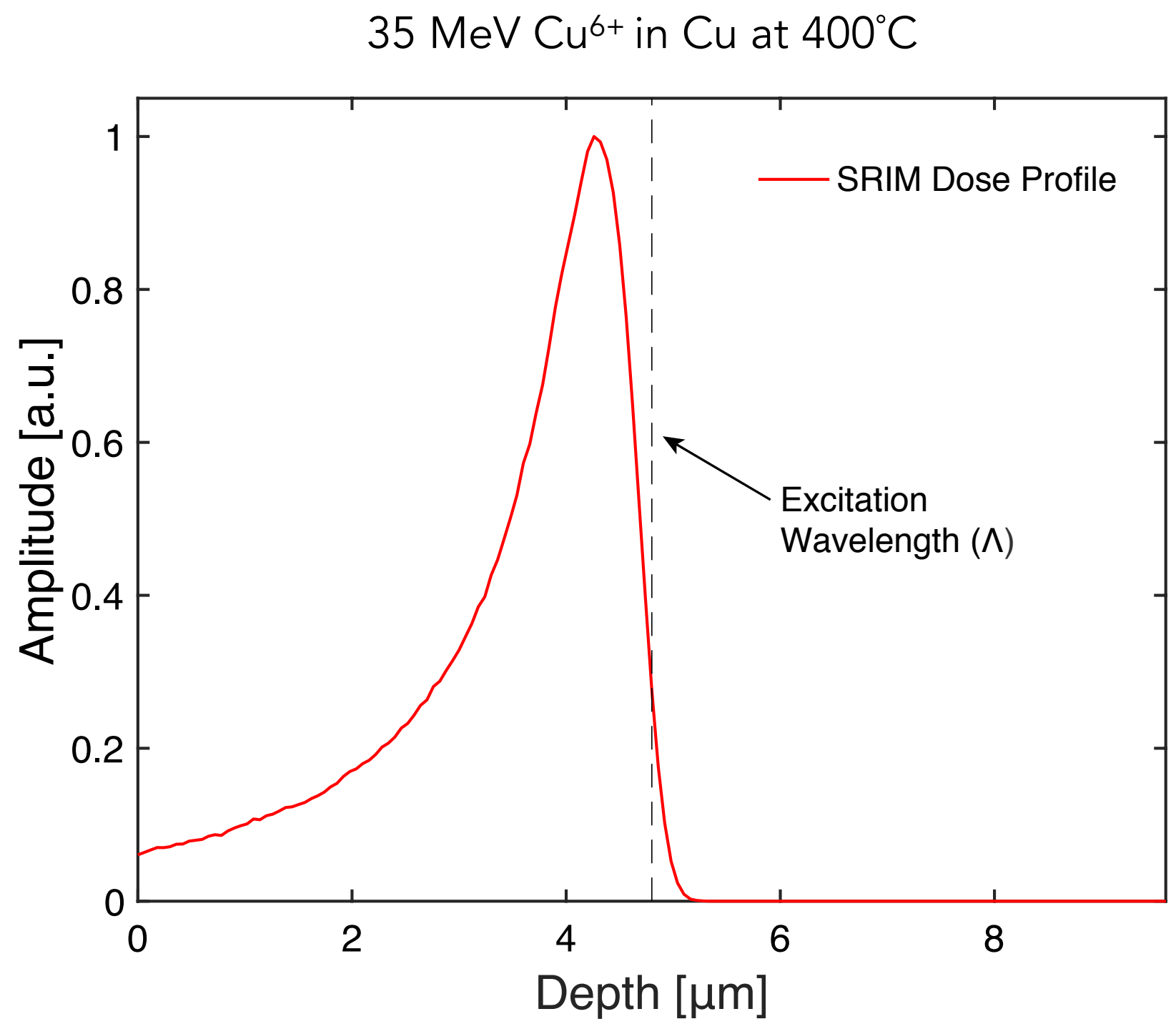


$35 \mathrm{MeV} \mathrm{Cu}^{6+}$ in $\mathrm{Cu}$ at $400^{\circ} \mathrm{C}$

\begin{tabular}{|c|c|}
\hline material & $\begin{array}{c}\text { single crystal } \\
\{111\} \mathrm{Cu}\end{array}$ \\
\hline ion species & $\mathrm{Cu}^{6+}$ \\
\hline ion energy & $35 \mathrm{MeV}$ \\
\hline temperature & $400^{\circ} \mathrm{C}$ \\
\hline spot size & $0.19 \mathrm{~cm}^{2}$ \\
\hline $\begin{array}{c}\text { beam current } \\
\text { target doses } \\
\text { (peak) }\end{array}$ & $\begin{array}{l}0,5,10,30 \mathrm{nA} \\
50,100 \mathrm{dpa}\end{array}$ \\
\hline
\end{tabular}

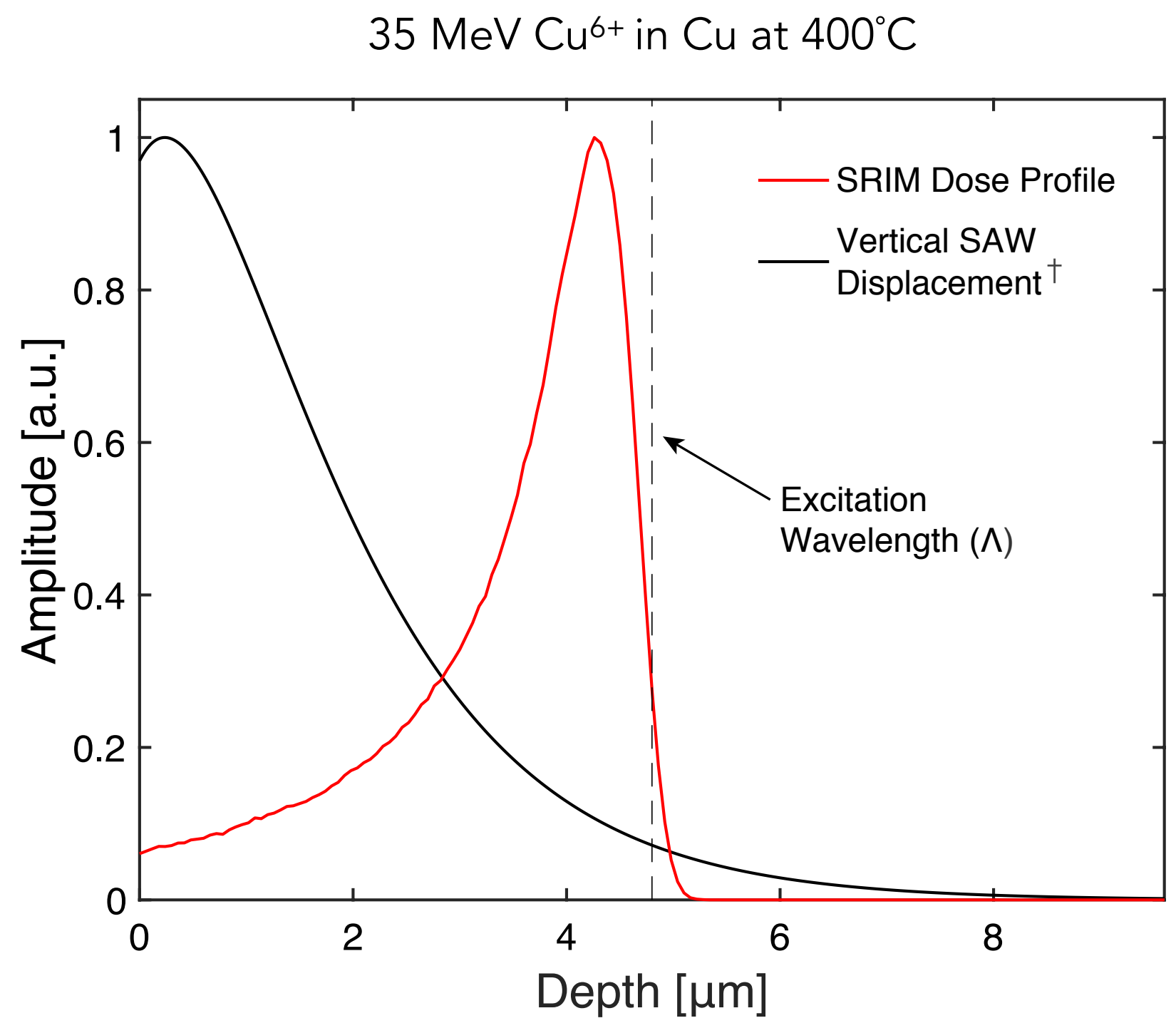




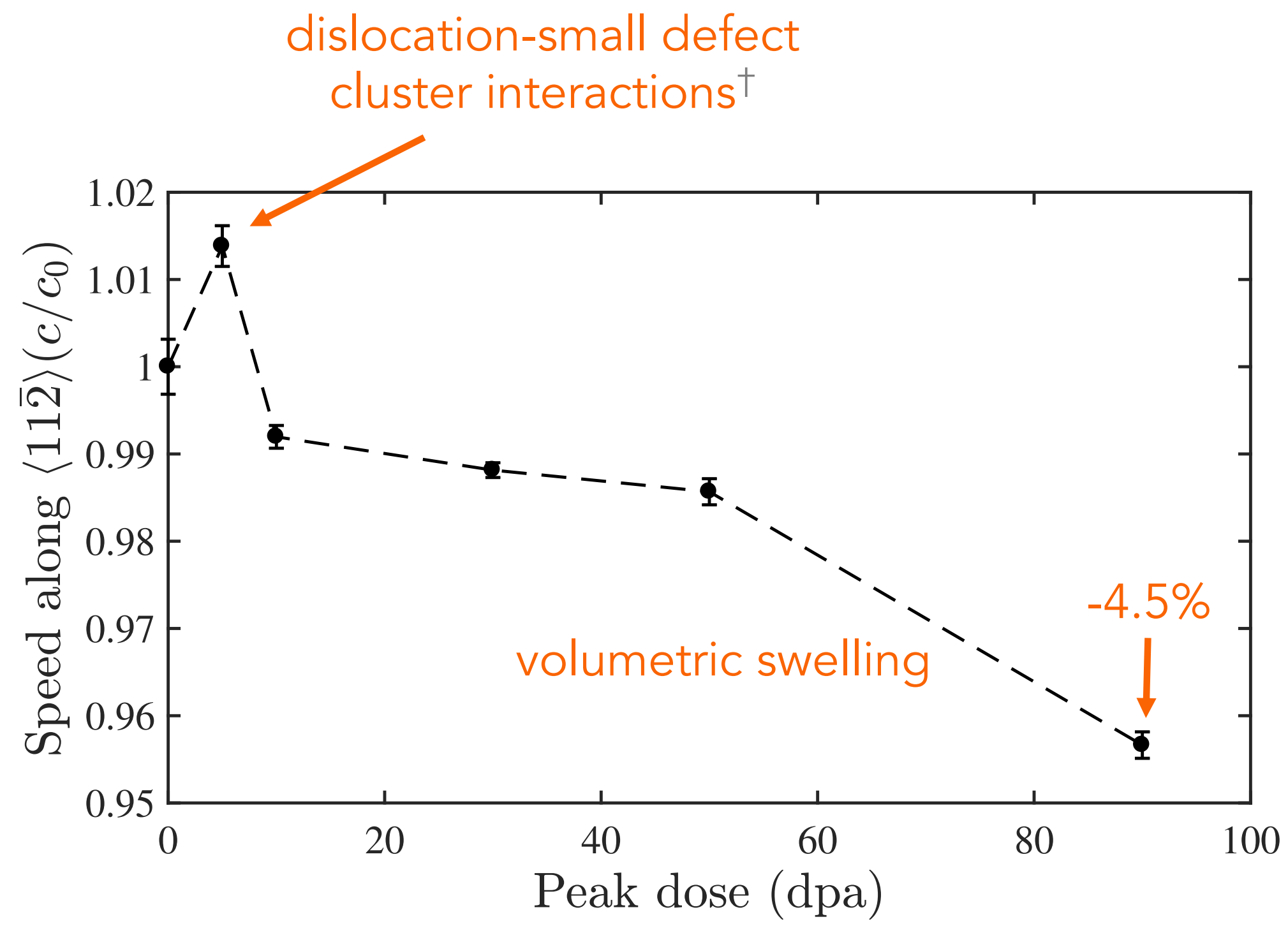




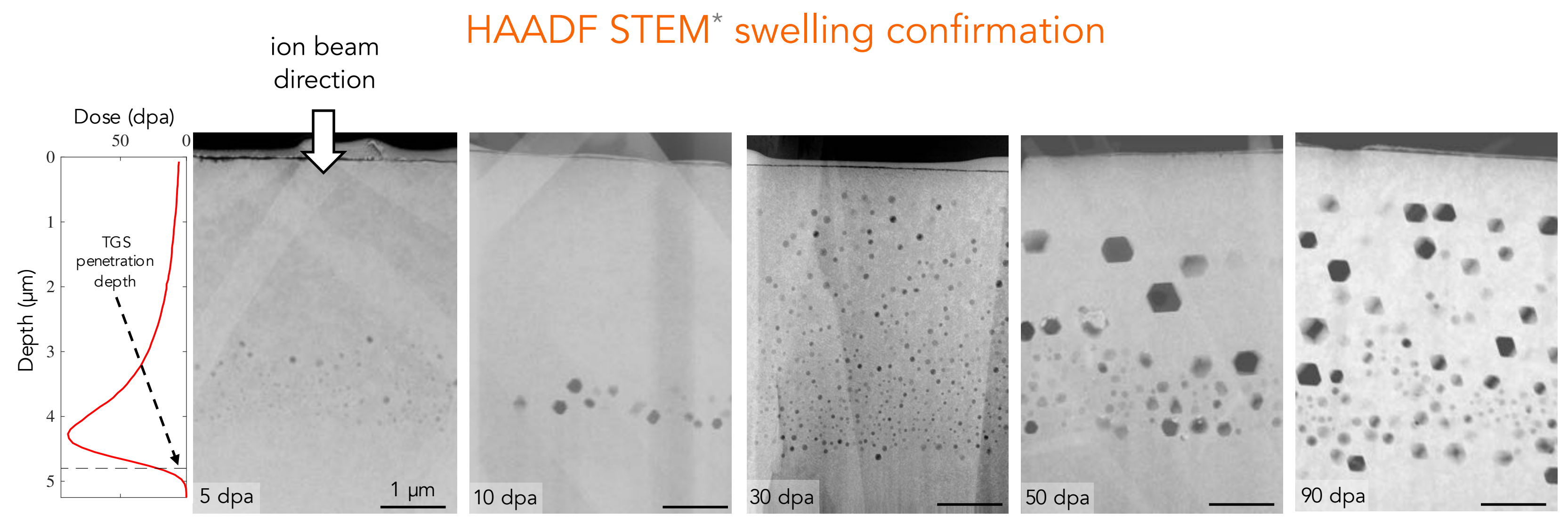
transmission electron microscopy 


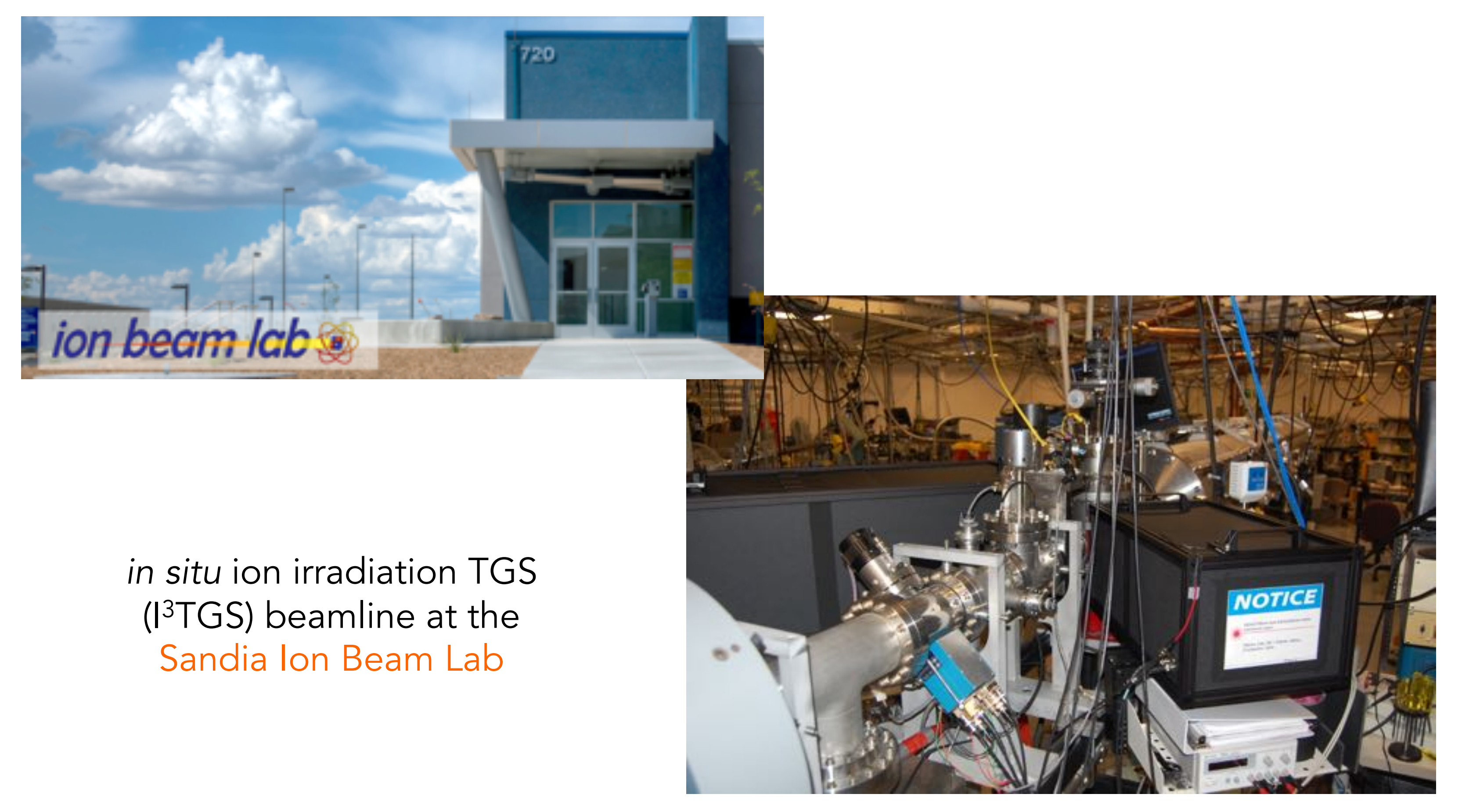




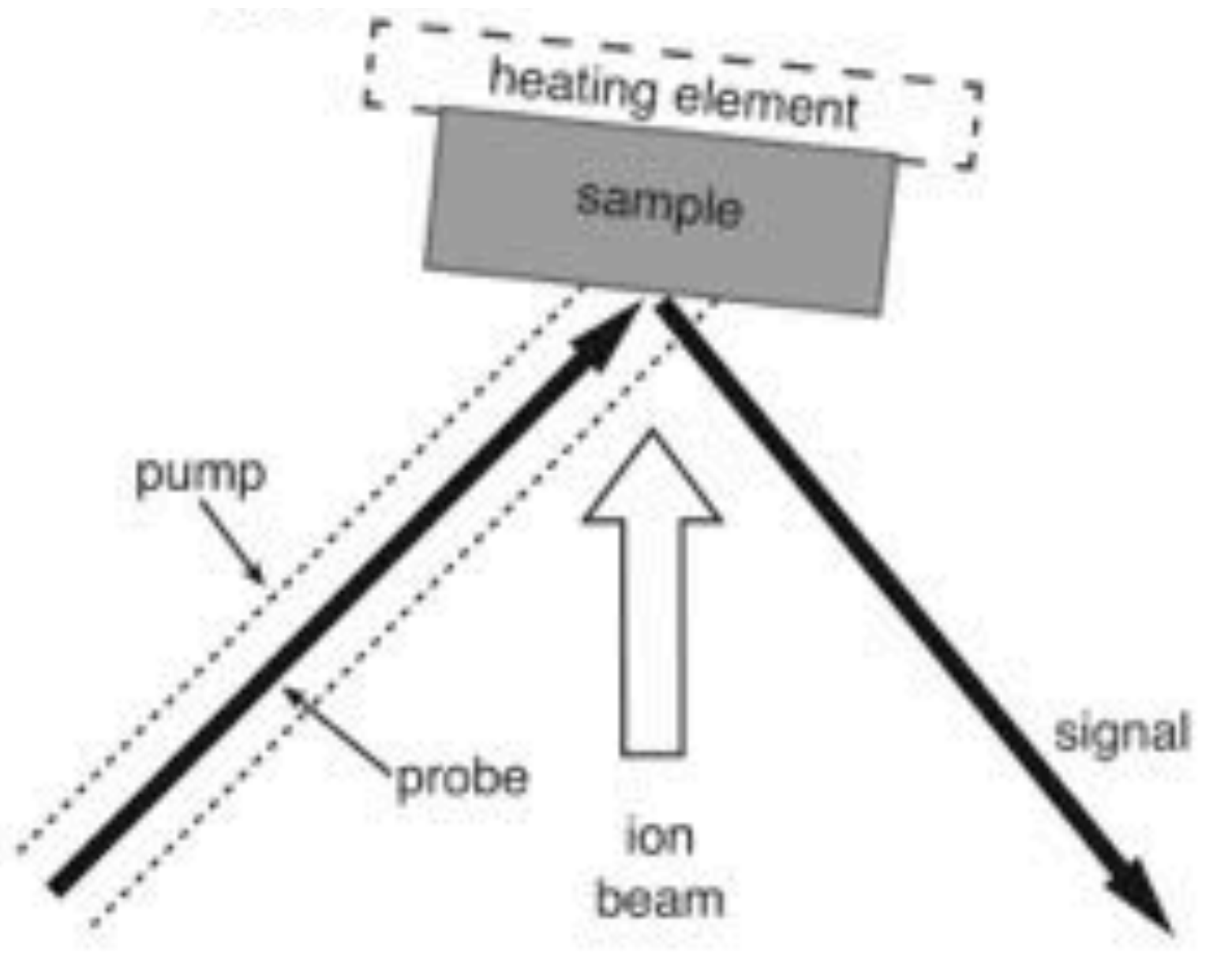

Dennett et al., NIMB 440 (2019) 126-138 doi: 10.1016/j.nimb.2018.10.025

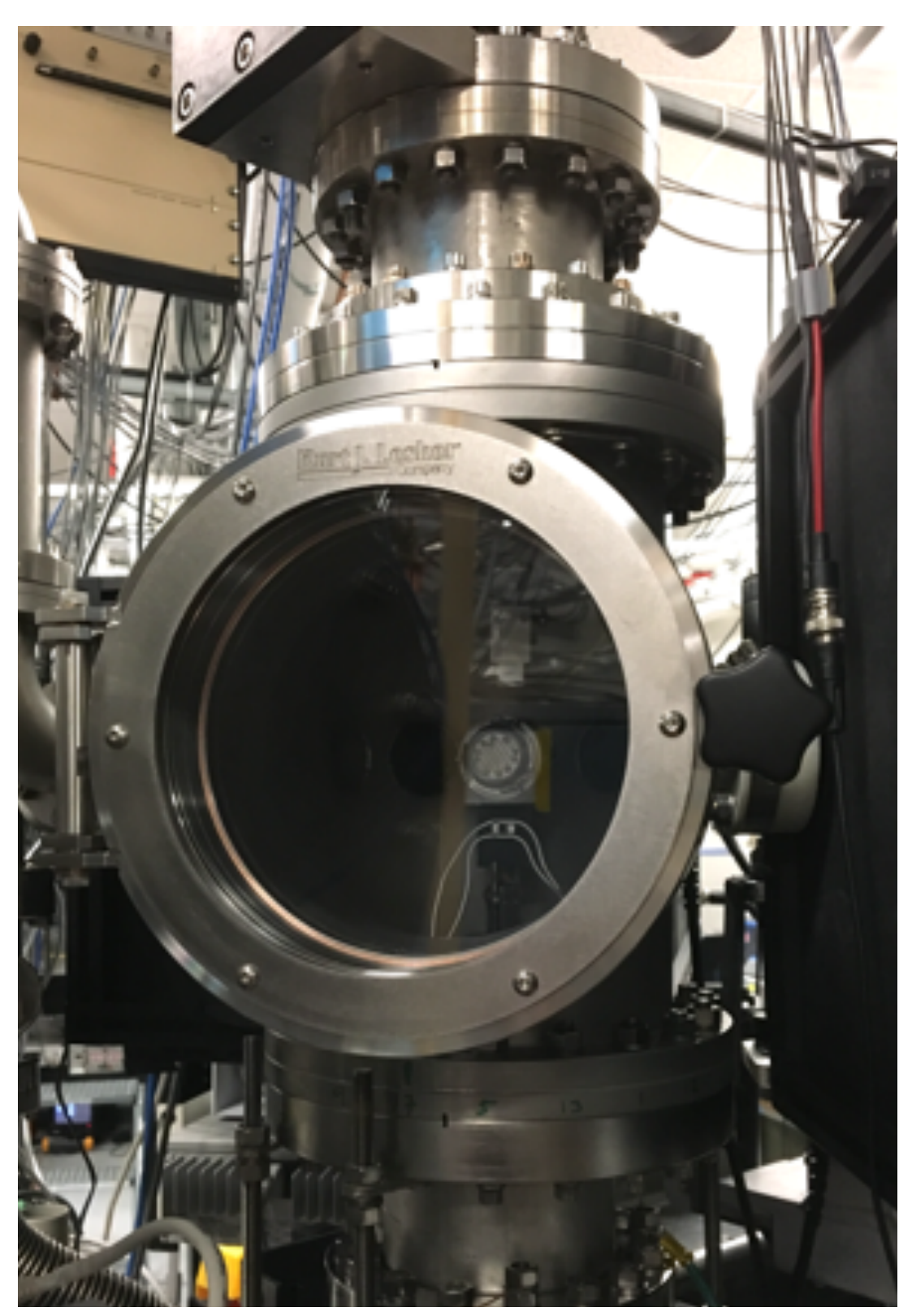

I3TGS target chamber 
- high strength

- thermal stability

- wear resistance

- corrosion resistance

- irradiation tolerance
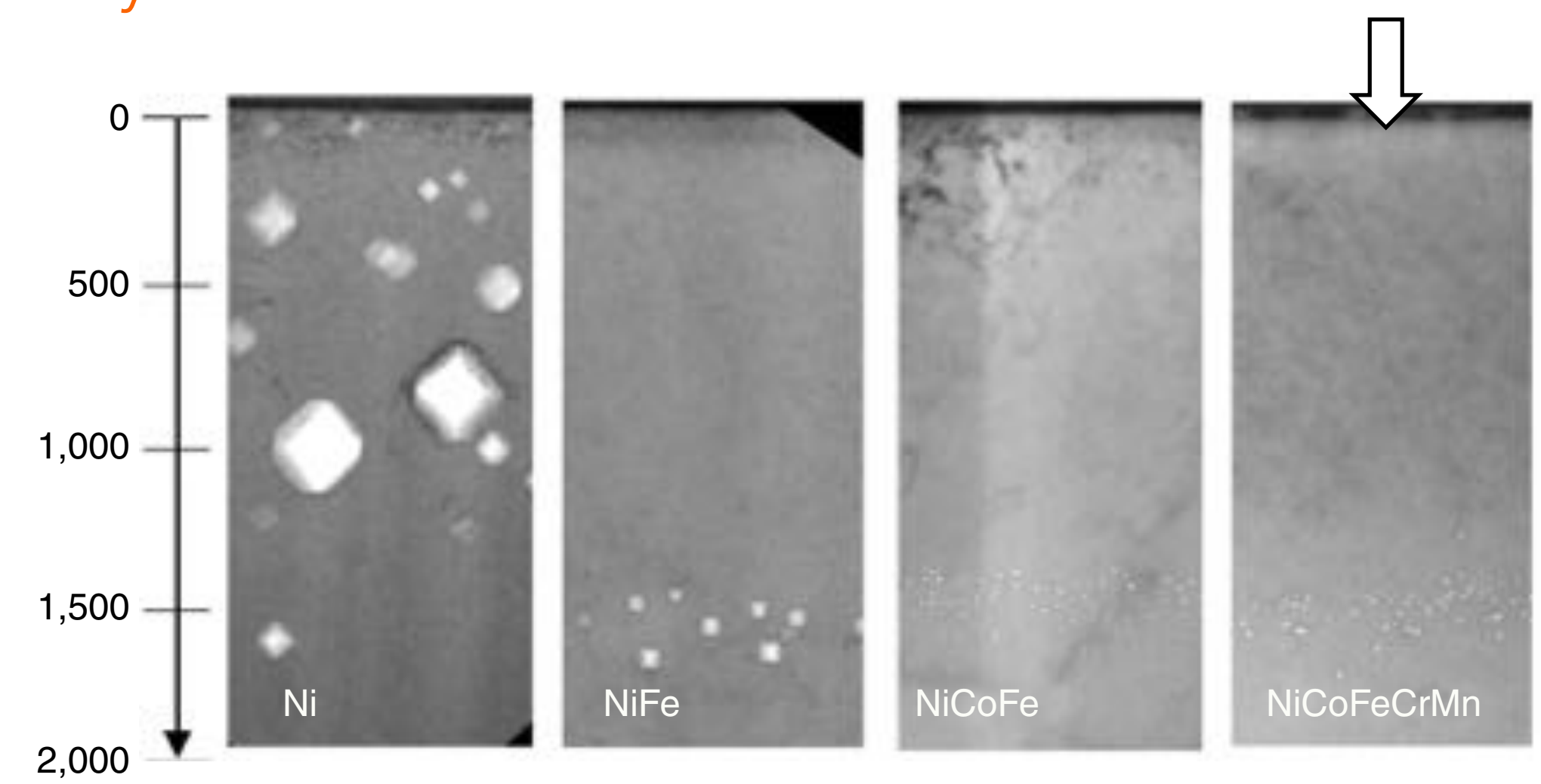

bright-field TEM of alloys irradiated with $1.5 \mathrm{MeV}$ $\mathrm{Ni}^{+}$to $60 \mathrm{dpa}$ peak at $500^{\circ} \mathrm{C}$ 


\begin{tabular}{|c|c|c|}
\hline $\begin{array}{c}\text { equiatomic } \\
\text { composition }\end{array}$ & $\begin{array}{c}\text { single/ } \\
\text { polycrystal }\end{array}$ & source \\
\hline $\mathrm{Ni}$ & $\mathrm{SC}\{001\}$ & commercial \\
\hline $\mathrm{NiFe}$ & $\mathrm{SC}\{001\}$ & ORNL \\
\hline $\mathrm{NiCoCr}$ & $\mathrm{SC}\{001\}$ & ORNL \\
\hline $\mathrm{NiFeCoCr}$ & $\mathrm{SC}\{001\}$ & ORNL \\
\hline $\mathrm{NiFeCoCrMn}$ & polycrystal & ORNL \\
\hline
\end{tabular}

in situ long-timescale irradiation test matrix 


\begin{tabular}{|c|c|}
\hline surface angle & $<100>\{001\}$ \\
\hline ion species & $\mathrm{Ni}^{5+}$ \\
\hline ion energy & $31 \mathrm{MeV}$ \\
\hline temperature & $550^{\circ} \mathrm{C}$ \\
\hline TGS wavelength & $4.55 \mu \mathrm{m}$ \\
\hline peak dose & $60 \mathrm{dpa}$ \\
\hline peak dose rate & $1.6-1.8$ \\
$\times 10^{-3} \mathrm{dpa} / \mathrm{s}$ \\
\hline measurement time & $35 \mathrm{sec}$ \\
\hline $\begin{array}{c}\text { measurement } \\
\text { interval }\end{array}$ & $60 \mathrm{sec}$ \\
\hline $\begin{array}{c}\text { exposure time } \\
\text { per sample }\end{array}$ & $9.5-10.5 \mathrm{hours}$ \\
\hline
\end{tabular}

\section{void swelling at high temperatures and high doses}

$31 \mathrm{MeV} \mathrm{Ni}^{5+}$ in $\mathrm{Ni}$ at $550^{\circ} \mathrm{C}$

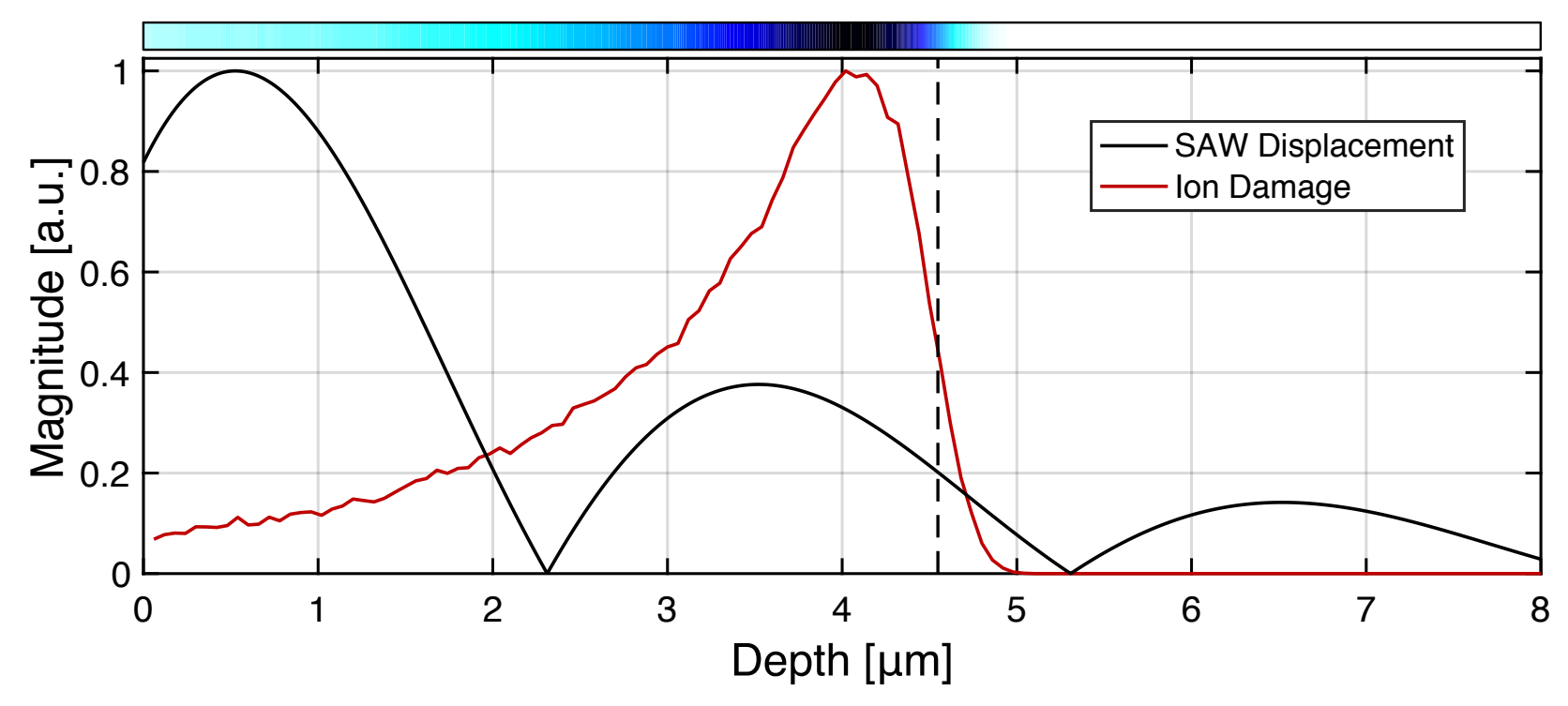


Irradiation Time [hr]

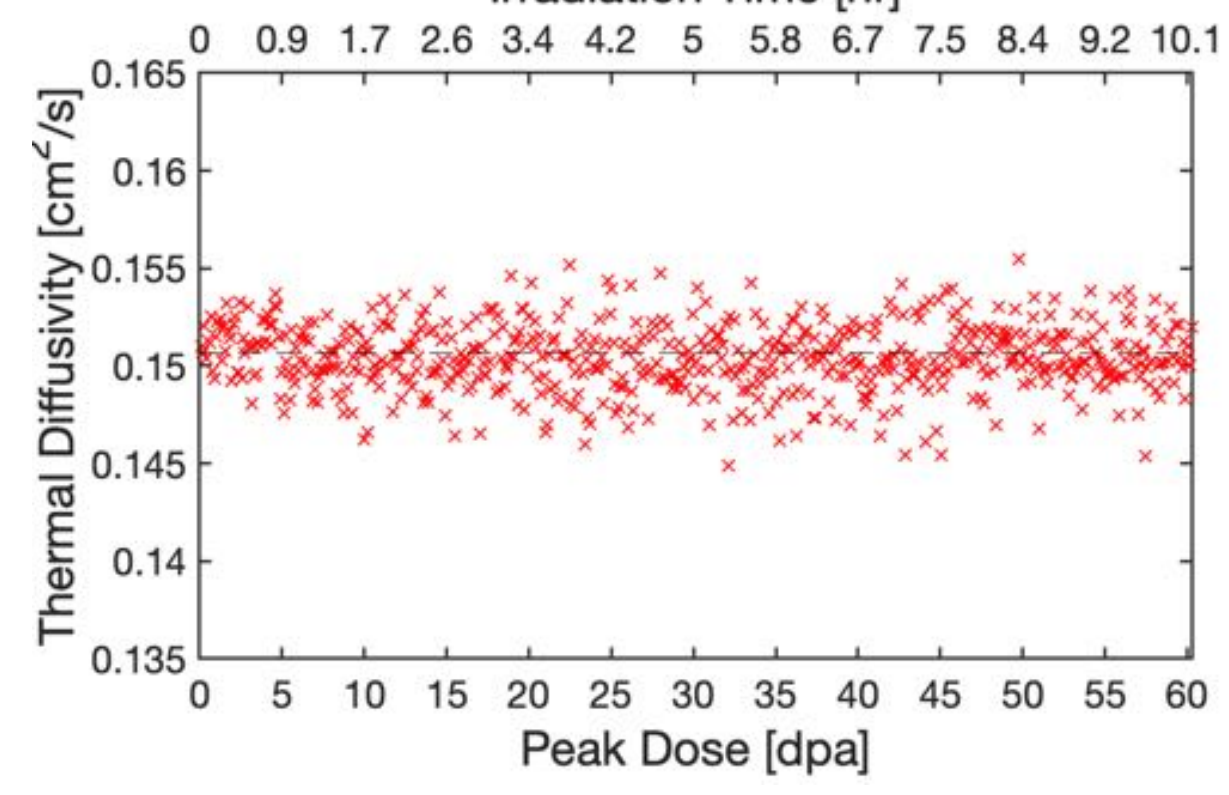

$\begin{array}{llllllllllll}0.9 & 1.7 & 2.6 & 3.4 & 4.2 & 5 & 5.8 & 6.7 & 7.5 & 8.4 & 9.2 & 10.1\end{array}$

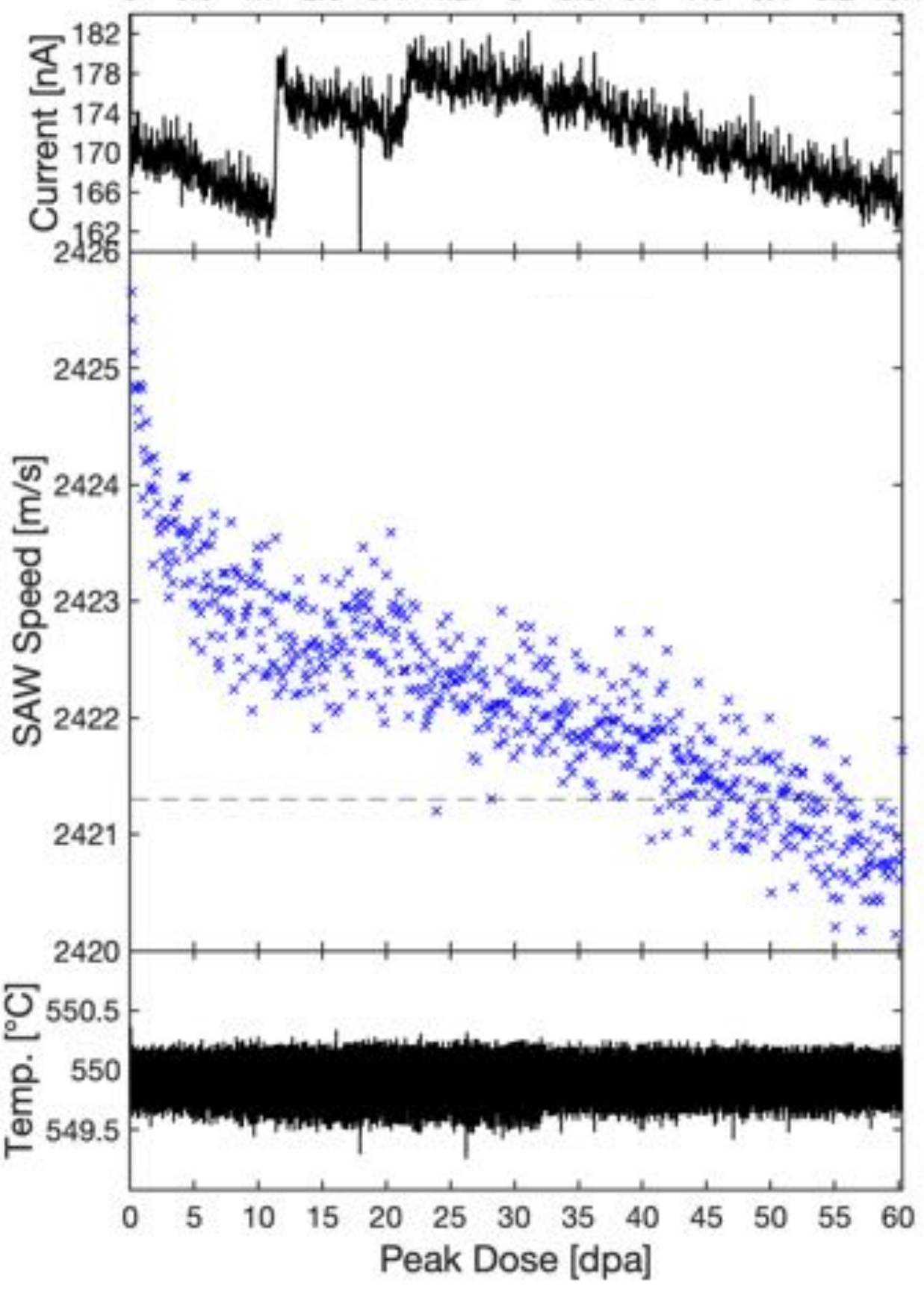


Irradiation Time [hr]

$\begin{array}{lllllllllllll}0 & 0.8 & 1.6 & 2.4 & 3.1 & 3.9 & 4.7 & 5.5 & 6.3 & 7.1 & 7.8 & 8.6 & 9.4\end{array}$

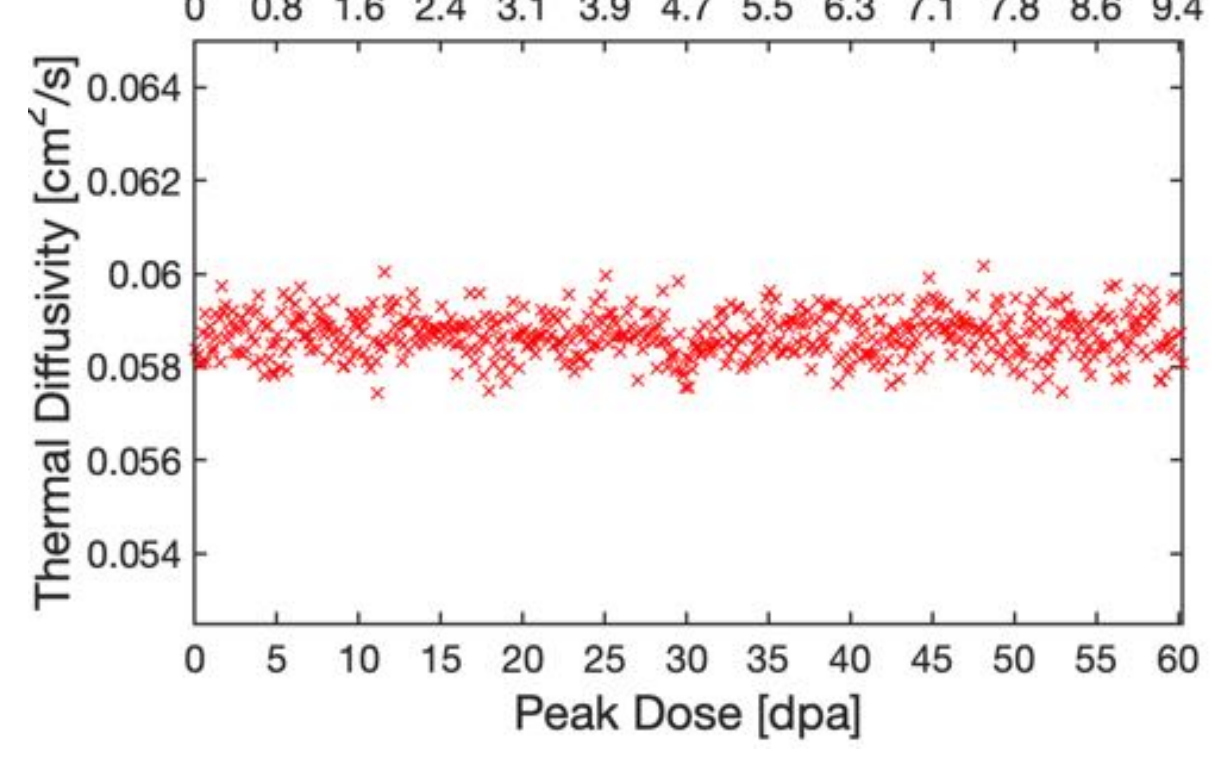

$\begin{array}{llllllllllllll}0 & 0.8 & 1.6 & 2.4 & 3.1 & 3.9 & 4.7 & 5.5 & 6.3 & 7.1 & 7.8 & 8.6 & 9.4\end{array}$

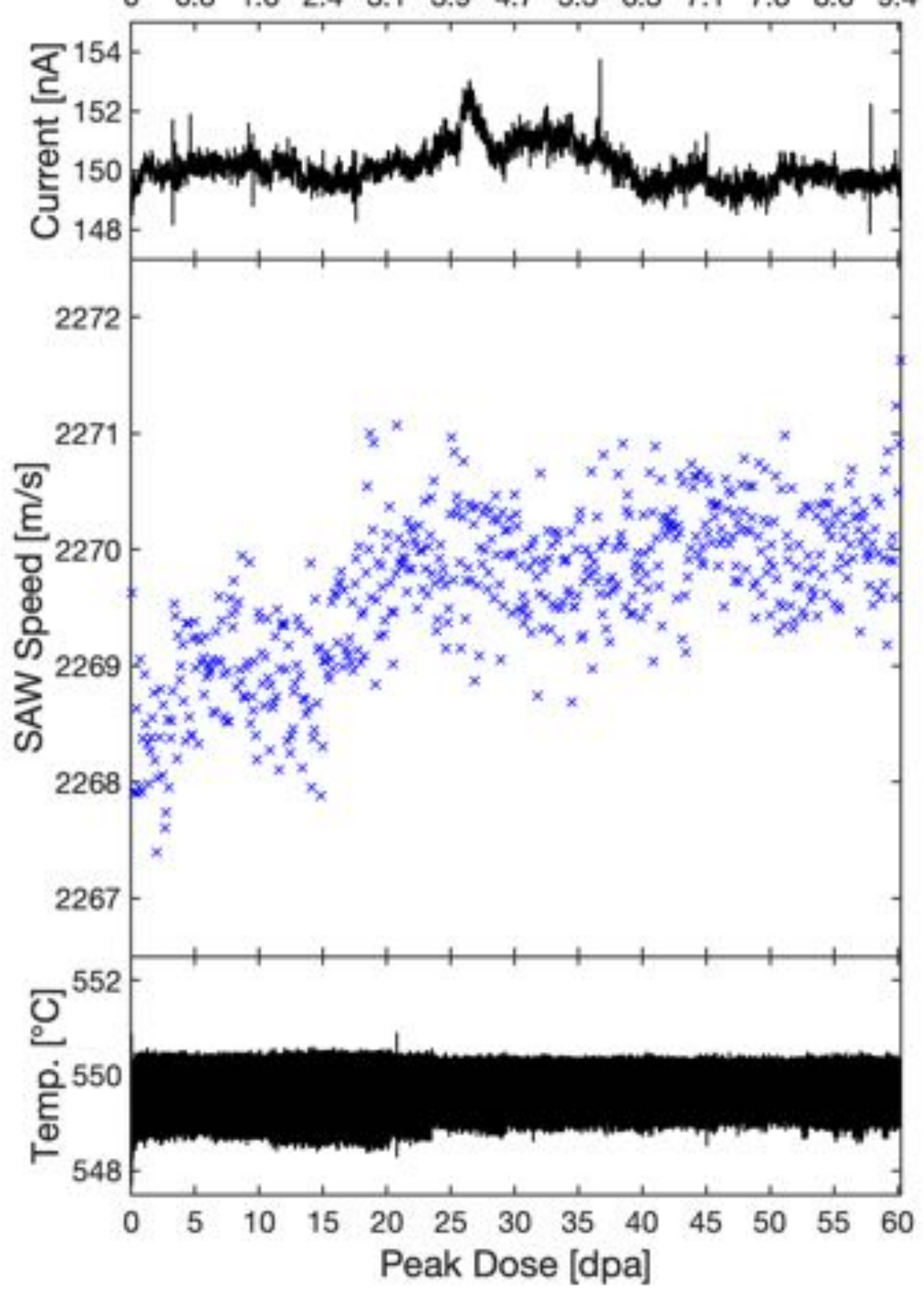




\section{$\mathrm{NiCoCr}$}

Irradiation Time [hr]

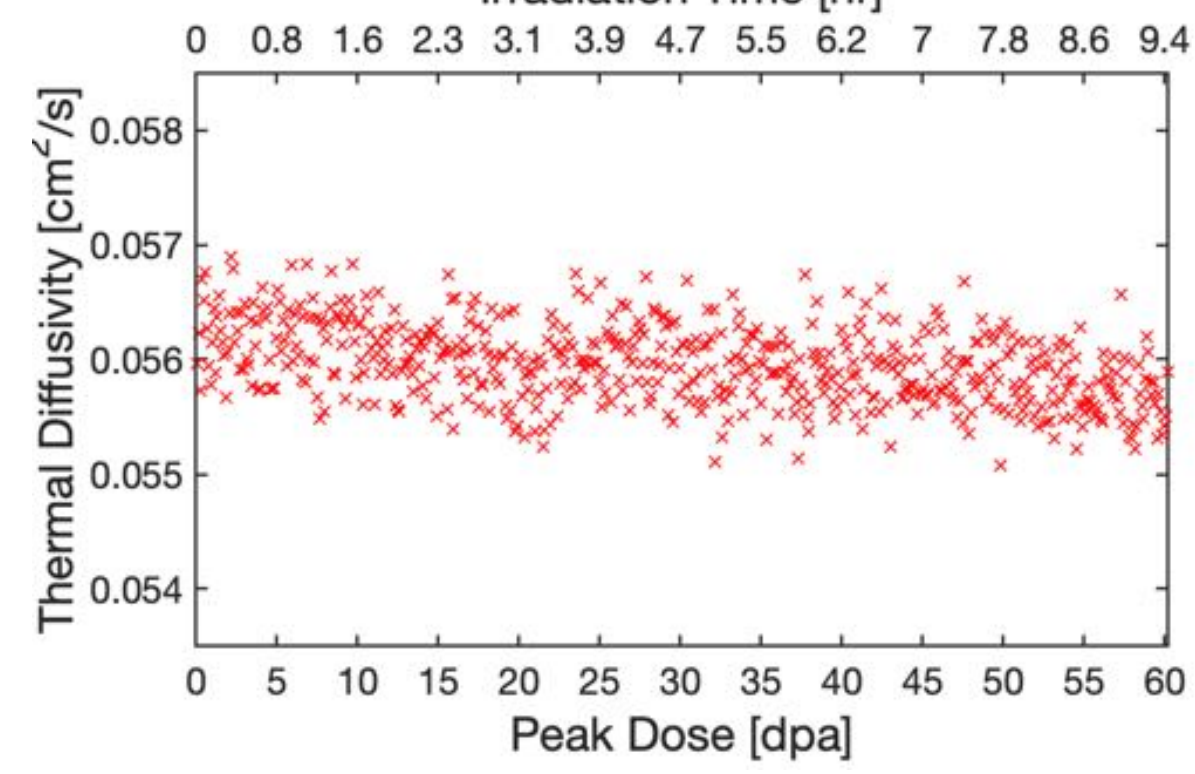

$\begin{array}{llllllllllll}0.8 & 1.6 & 2.3 & 3.1 & 3.9 & 4.7 & 5.5 & 6.2 & 7 & 7.8 & 8.6 & 9.4\end{array}$

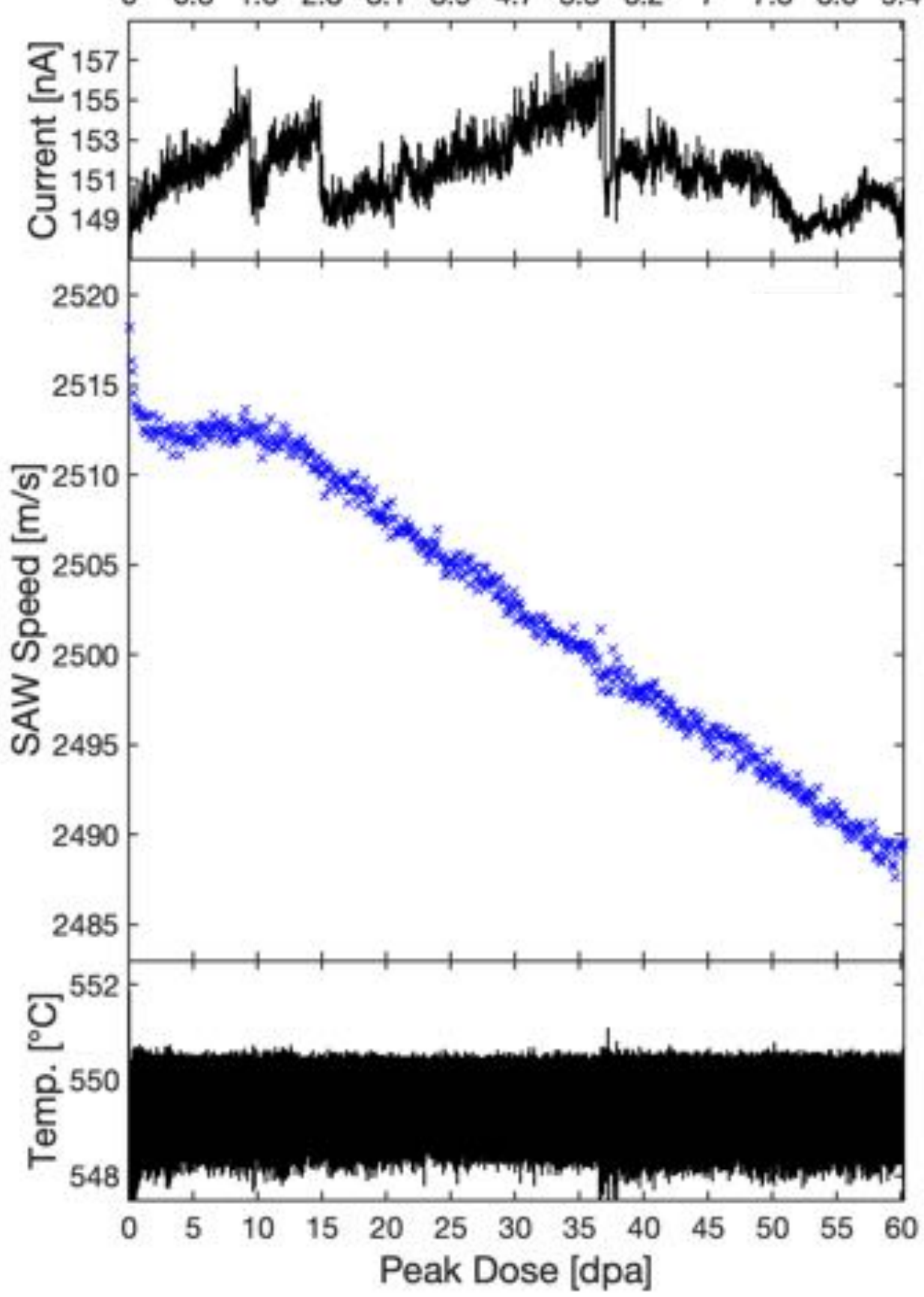




\section{$\mathrm{NiFeCoCr}$}

Irradiation Time [hr]

$\begin{array}{lllllllllllll}0 & 0.8 & 1.6 & 2.4 & 3.3 & 4.2 & 5.1 & 6 & 6.9 & 7.8 & 8.7 & 9.7 & 10.6\end{array}$

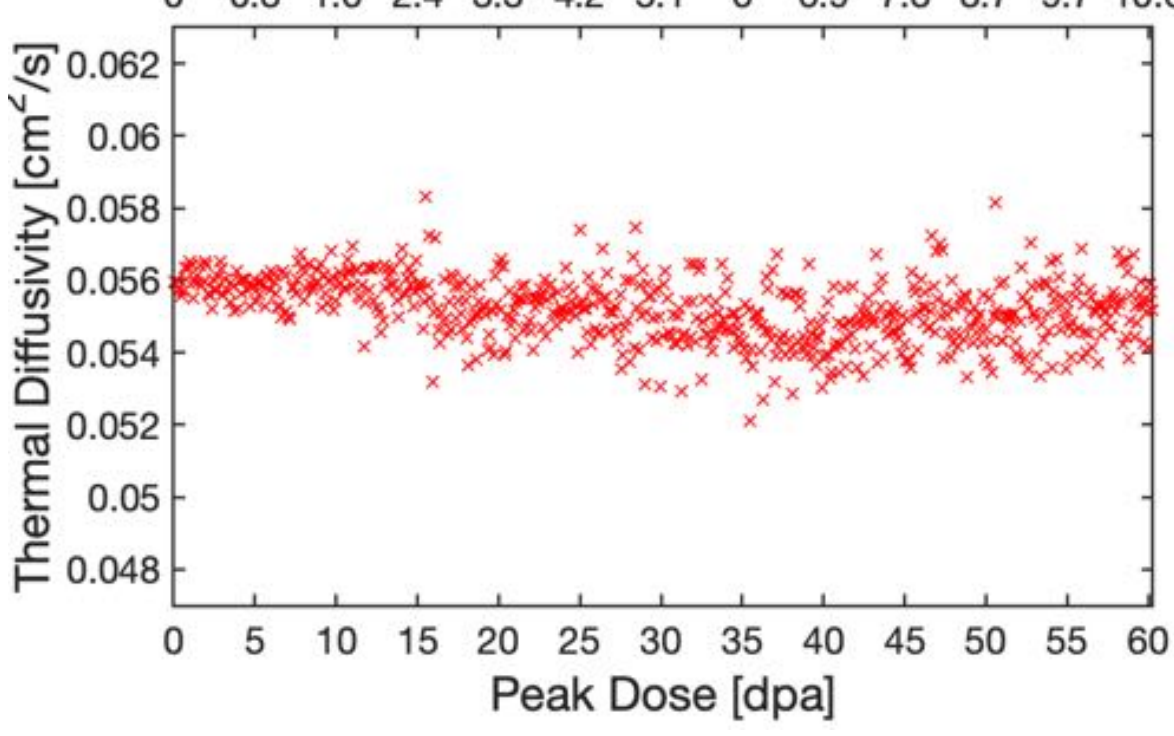

$\begin{array}{lllllllllllll}0 & 0.8 & 1.6 & 2.4 & 3.3 & 4.2 & 5.1 & 6 & 6.9 & 7.8 & 8.7 & 9.7 & 10.6\end{array}$

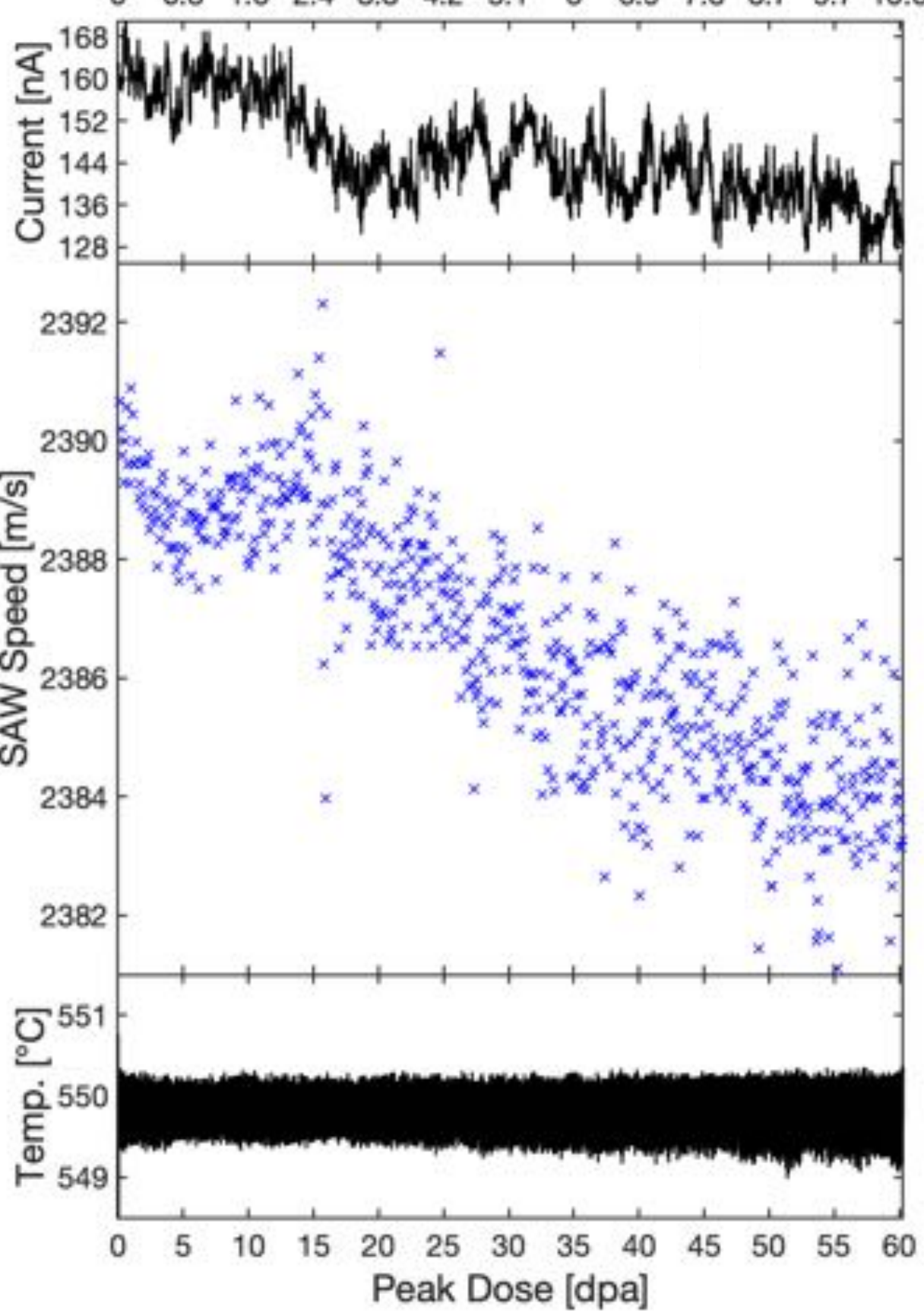




\section{$\mathrm{NiFeCoCrMn}$}
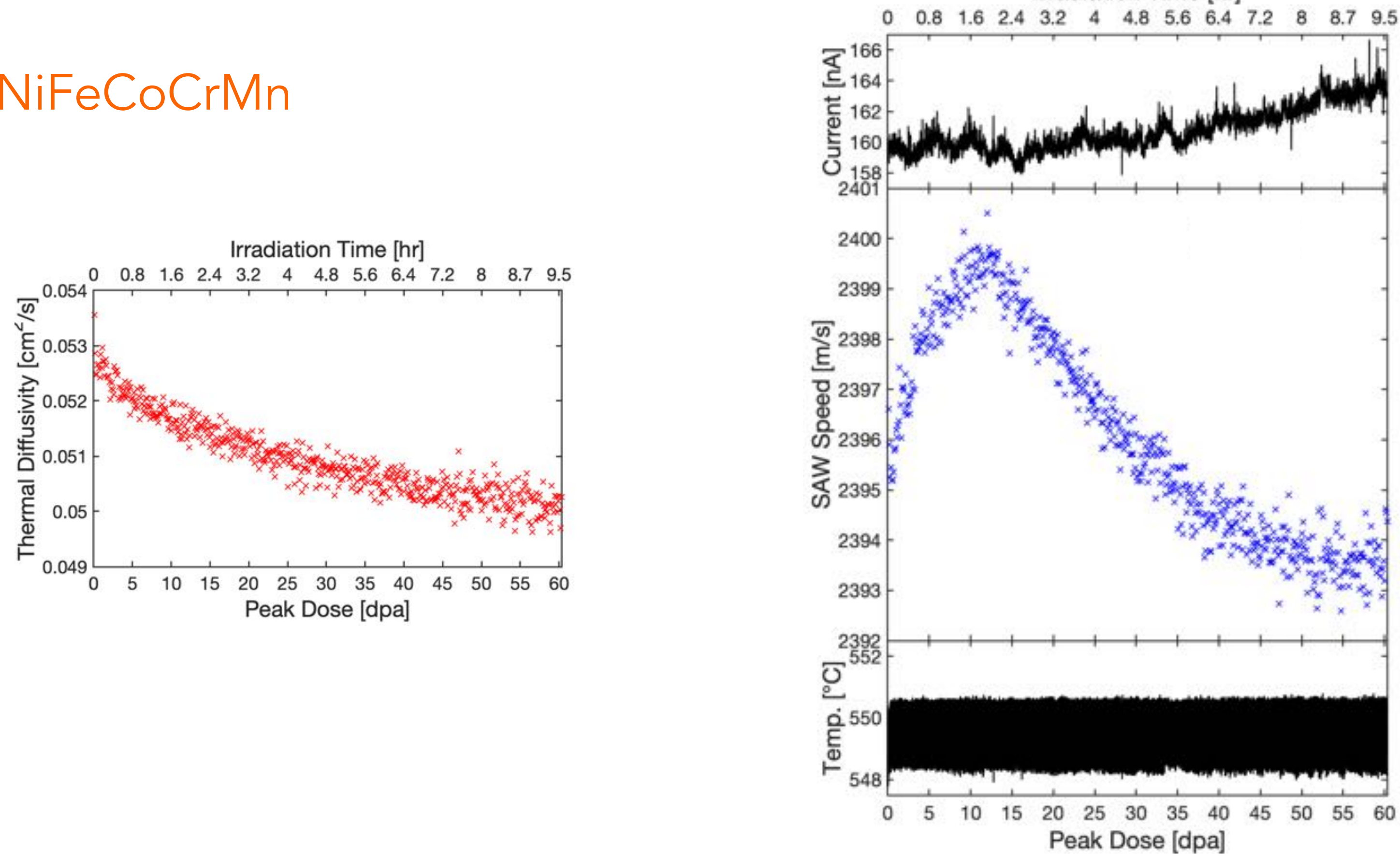


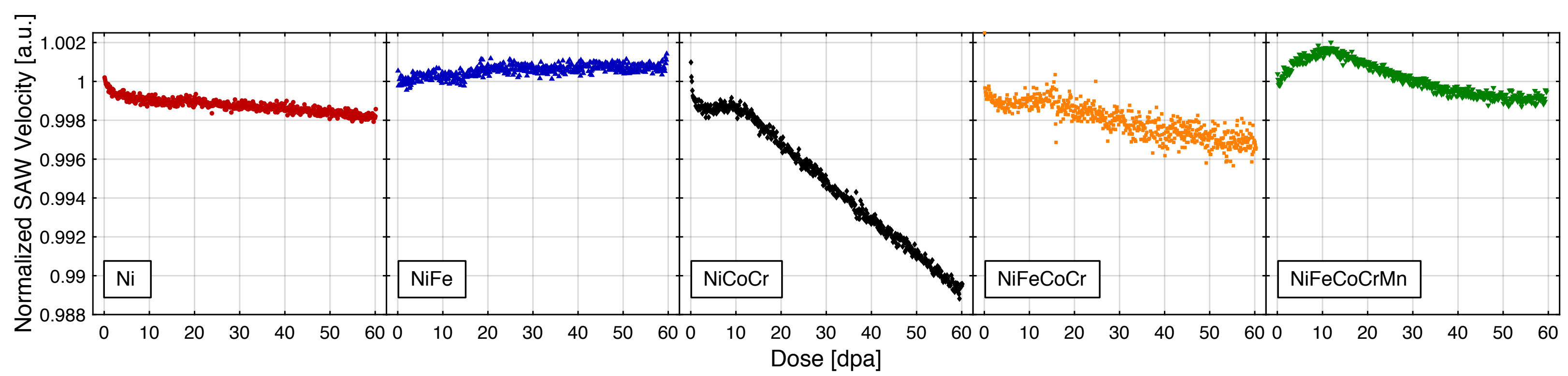

年
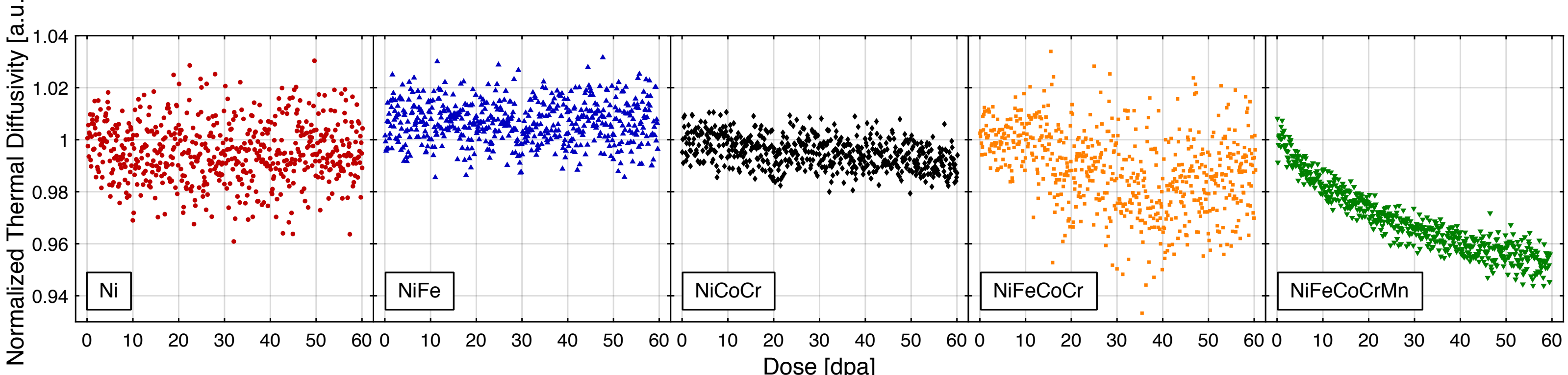


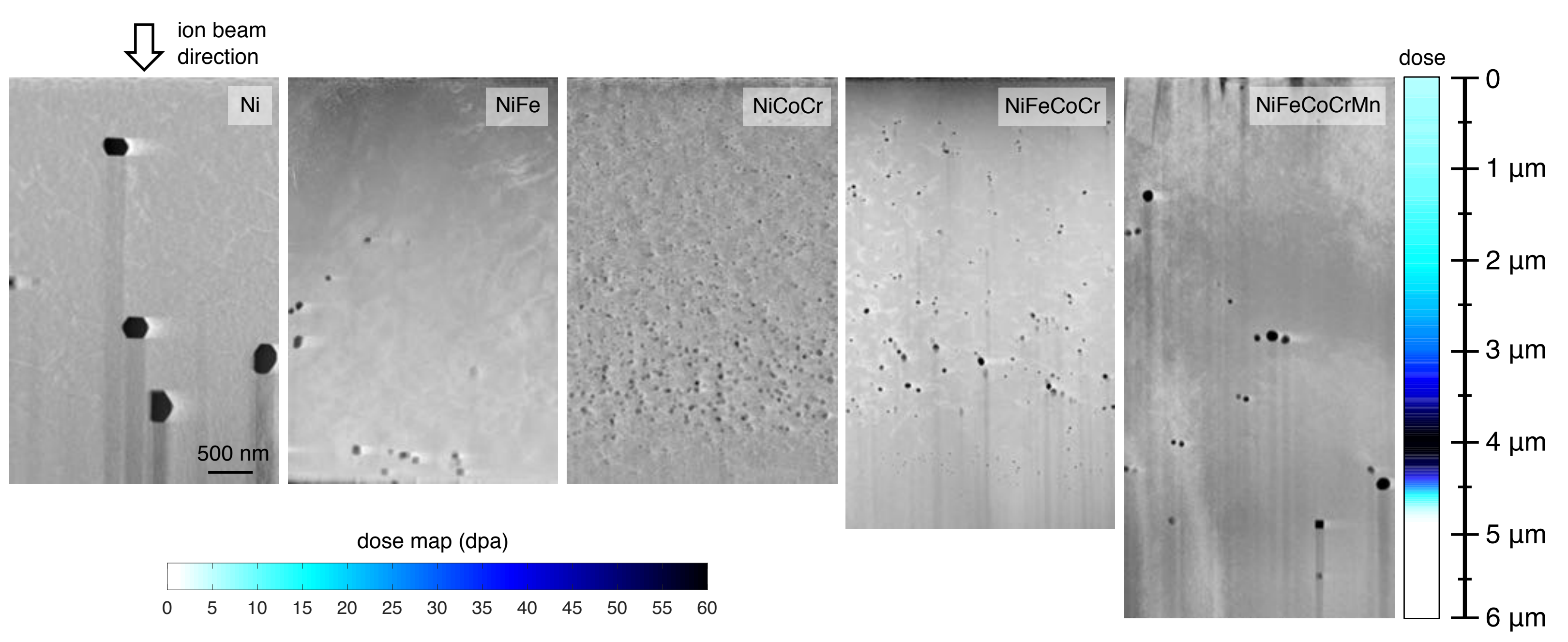



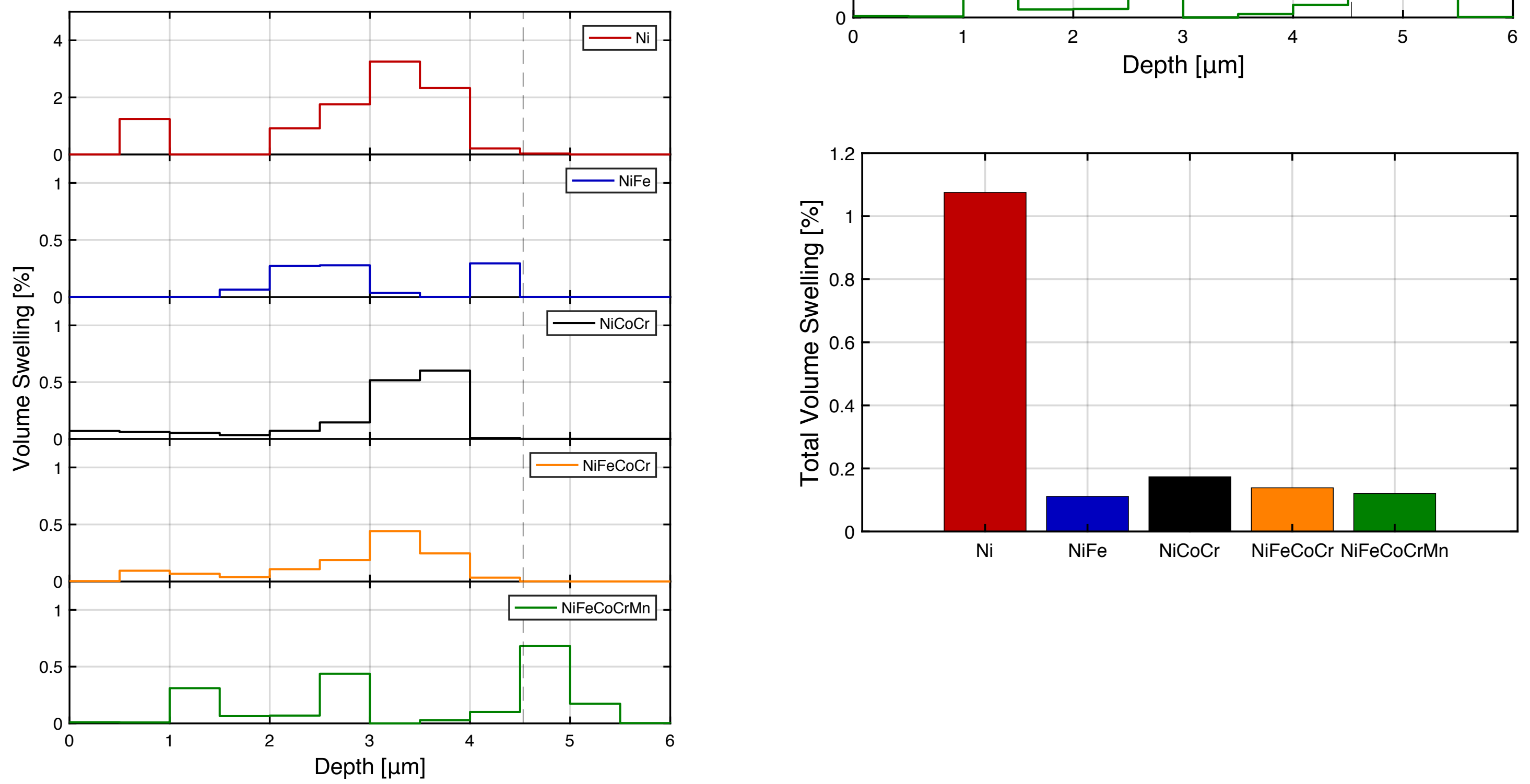
at temperature, ion beam off

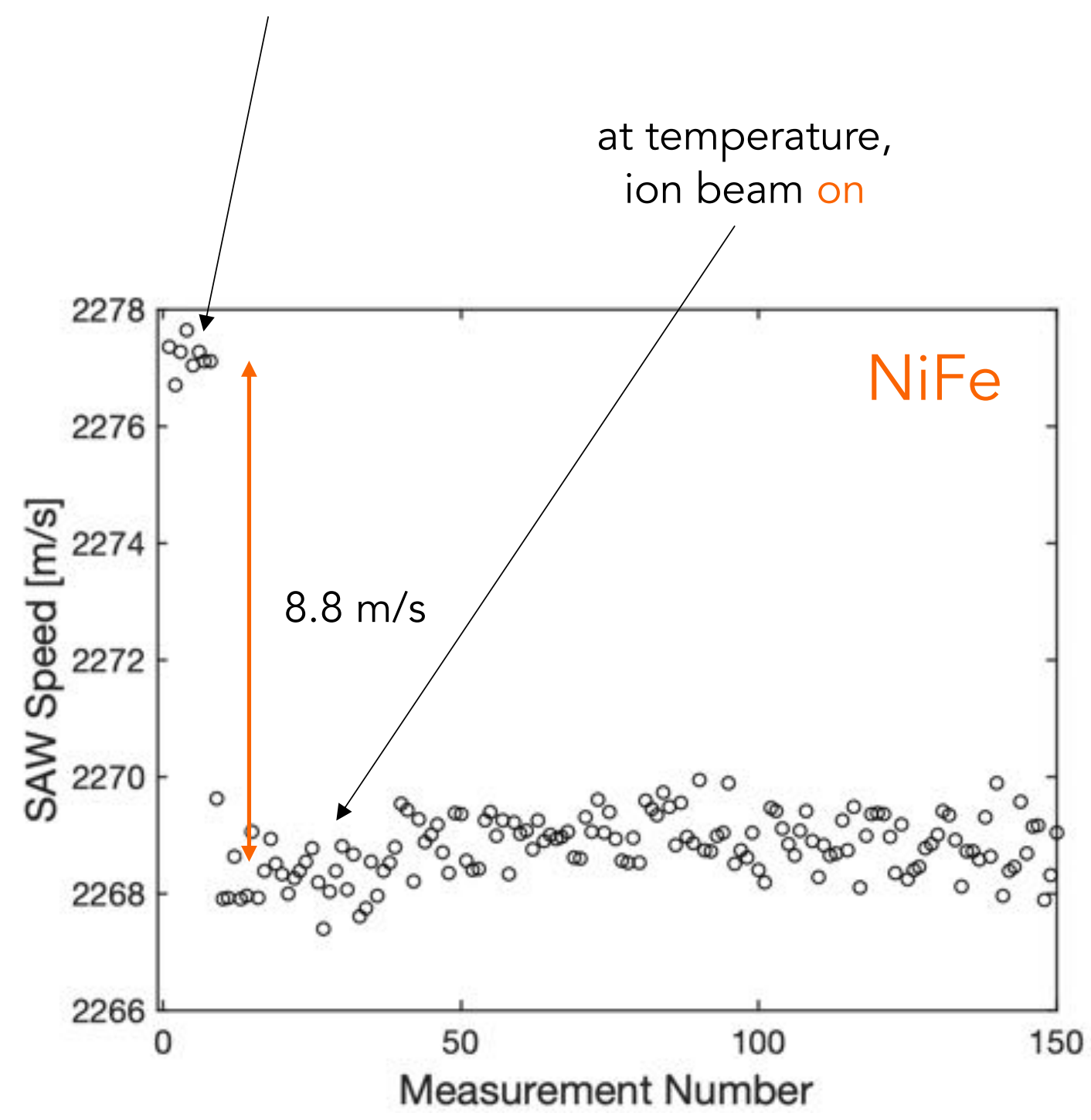

ionperature,

\section{'instantaneous' defect generation} affects mechanical properties

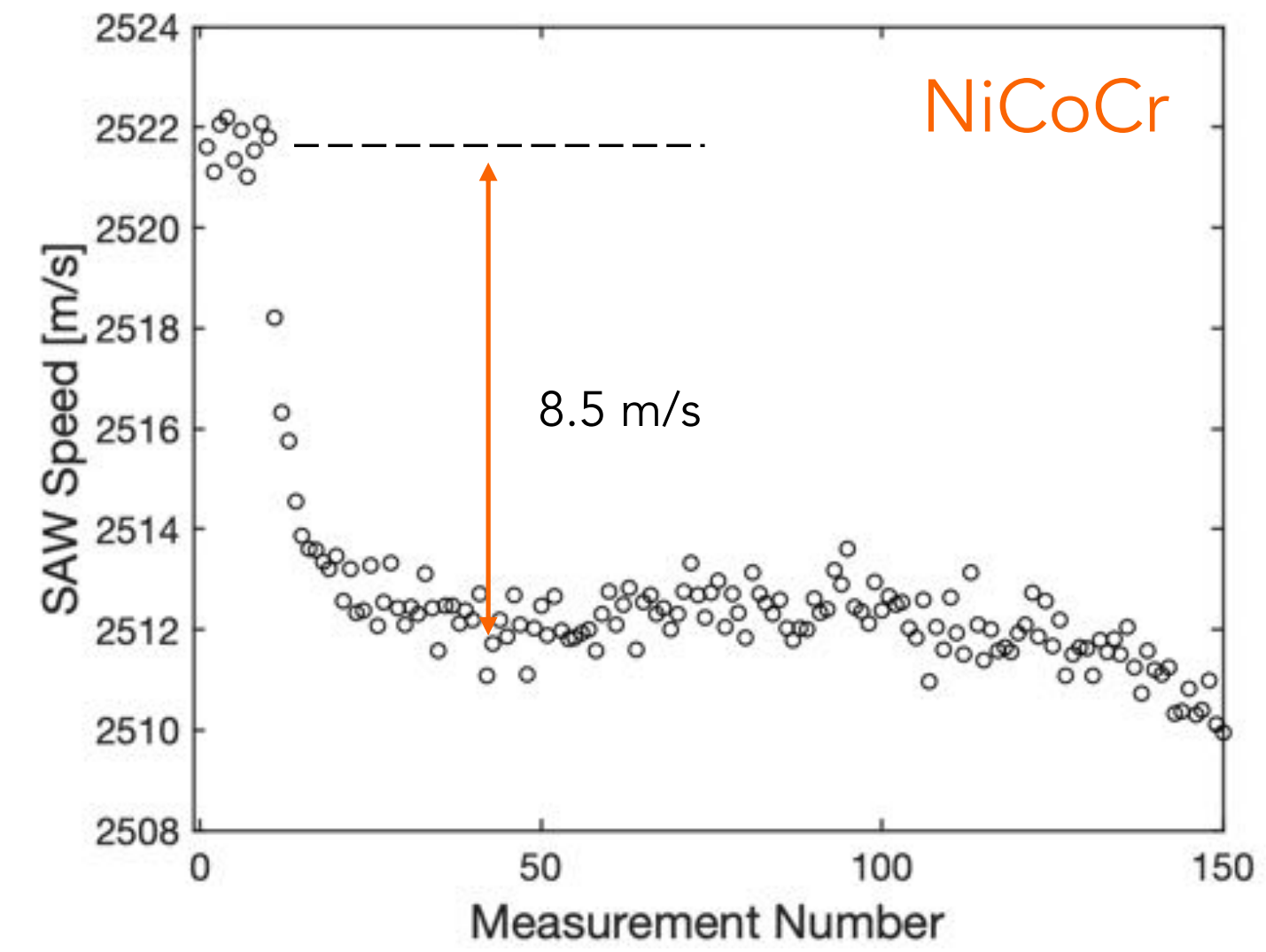




\begin{tabular}{|c|c|}
\hline surface angle & $<100>\{001\}$ \\
\hline ion species & $\mathrm{Ni}{ }^{6+}$ \\
\hline ion energy & $31 \mathrm{MeV}$ \\
\hline temperature & $500^{\circ} \mathrm{C}$ \\
\hline TGS wavelength & $4.55 \mu \mathrm{m}$ \\
\hline $\begin{array}{c}\text { dose rates } \\
\text { measurement } \\
\text { interval }\end{array}$ & $\begin{array}{c}1,5,10,20] \times \\
\mathrm{dpa} / \mathrm{s}\end{array}$ \\
\hline $\begin{array}{c}\text { annealing time } \\
\text { between impulses }\end{array}$ & $\sim 30 \mathrm{sec}$ \\
\hline
\end{tabular}

\section{instantaneous defect effects at varying dose rates}

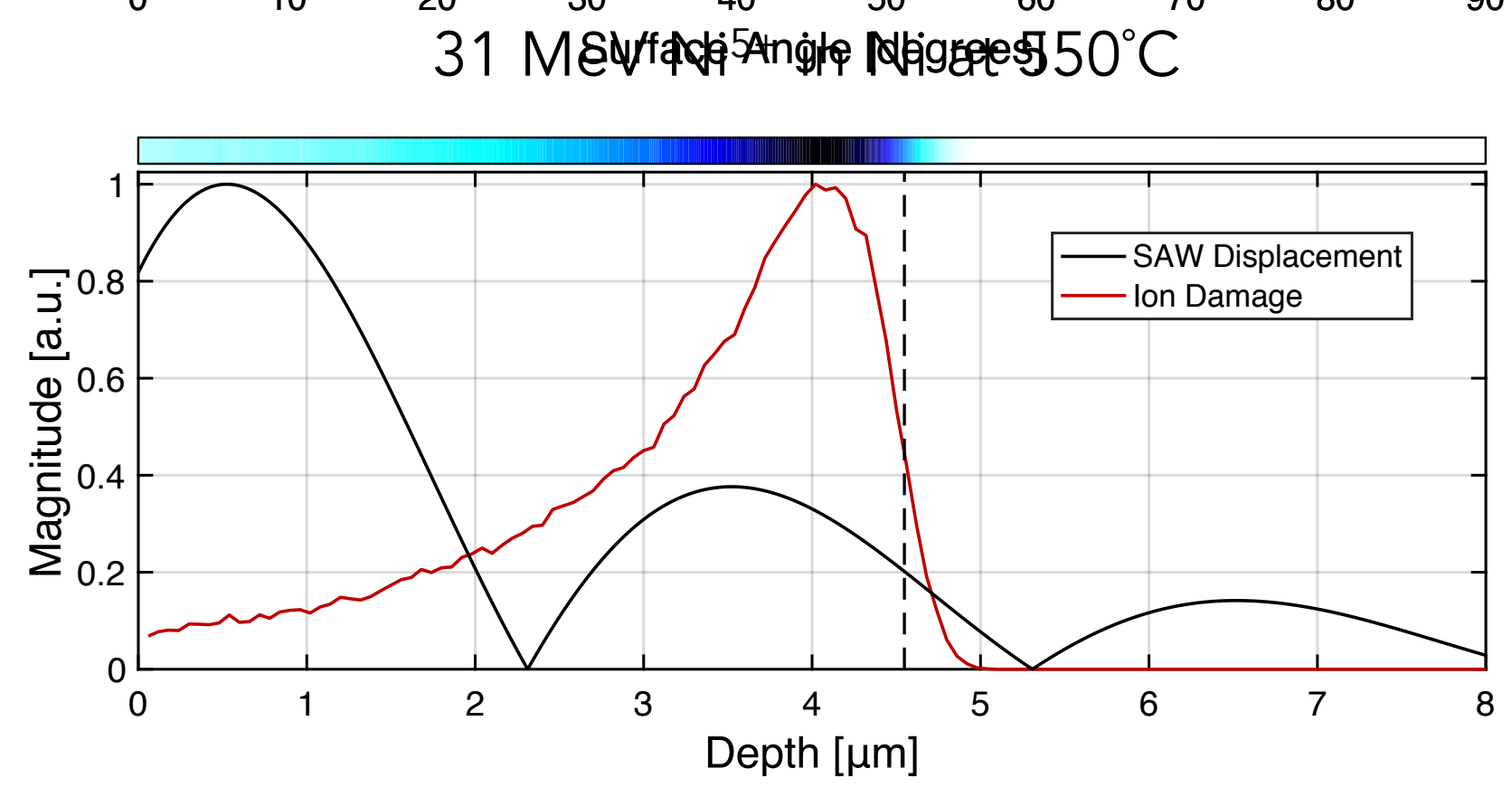




\section{example current and}

temperature records for $\mathrm{NiCoCr}$

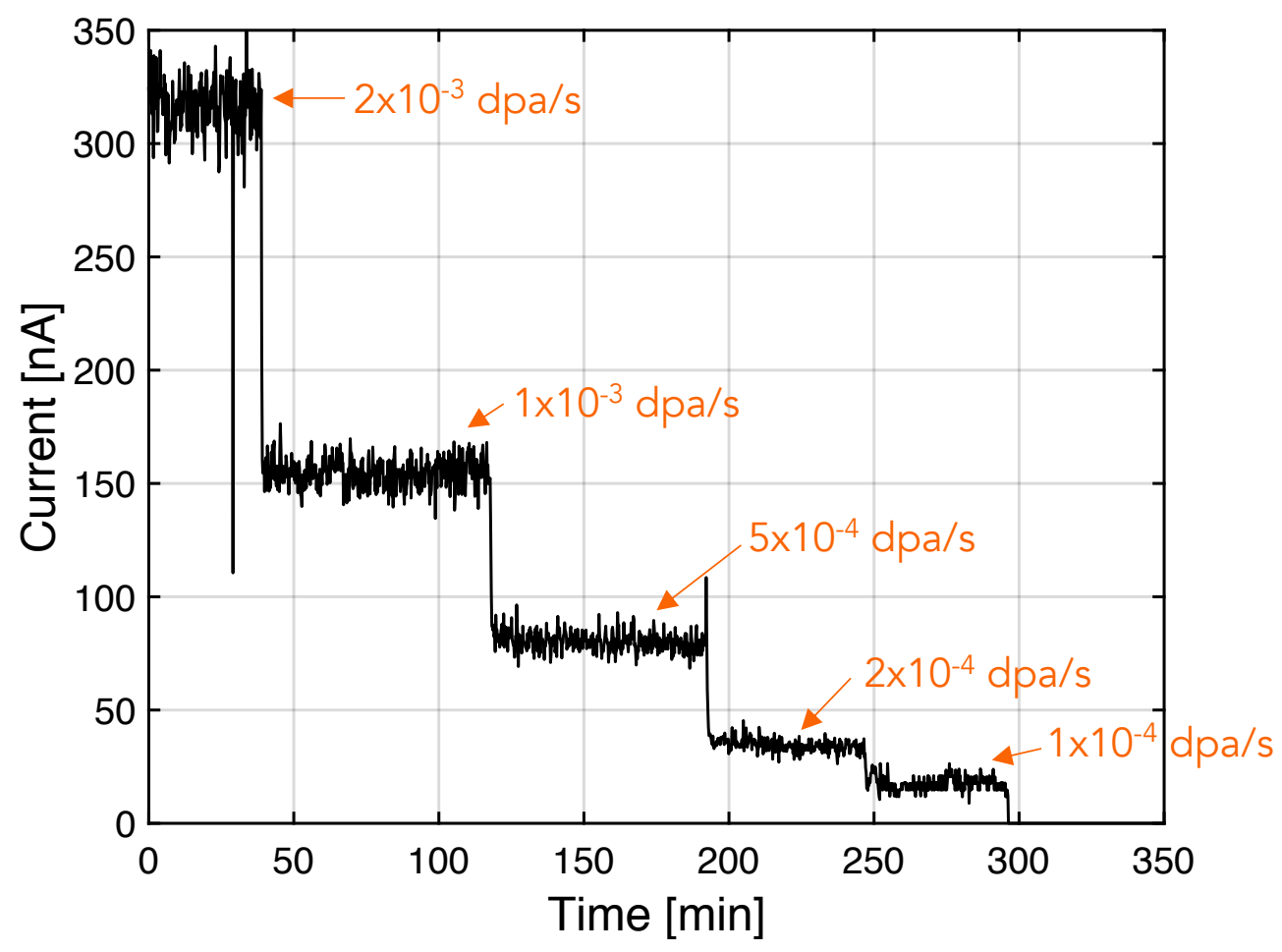

beam current lowered in steps between impulse experiments

higher beam heating and larger temperature fluctuations during high flux experiments

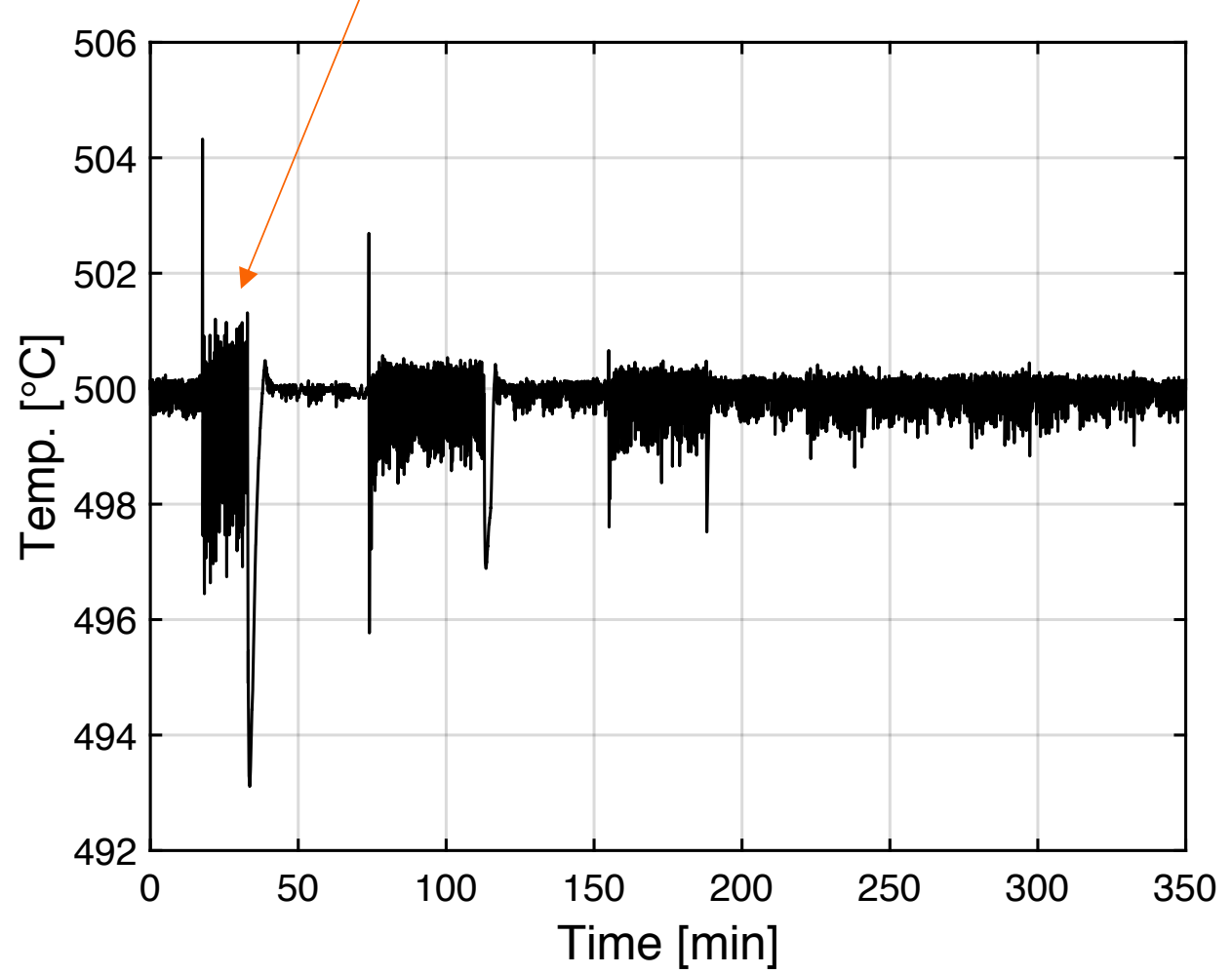




\section{Pure $\mathrm{Ni}$}

$1 \times 10^{-3} \mathrm{dpa} / \mathrm{s}$

$2 \times 10^{-4} \mathrm{dpa} / \mathrm{s}$

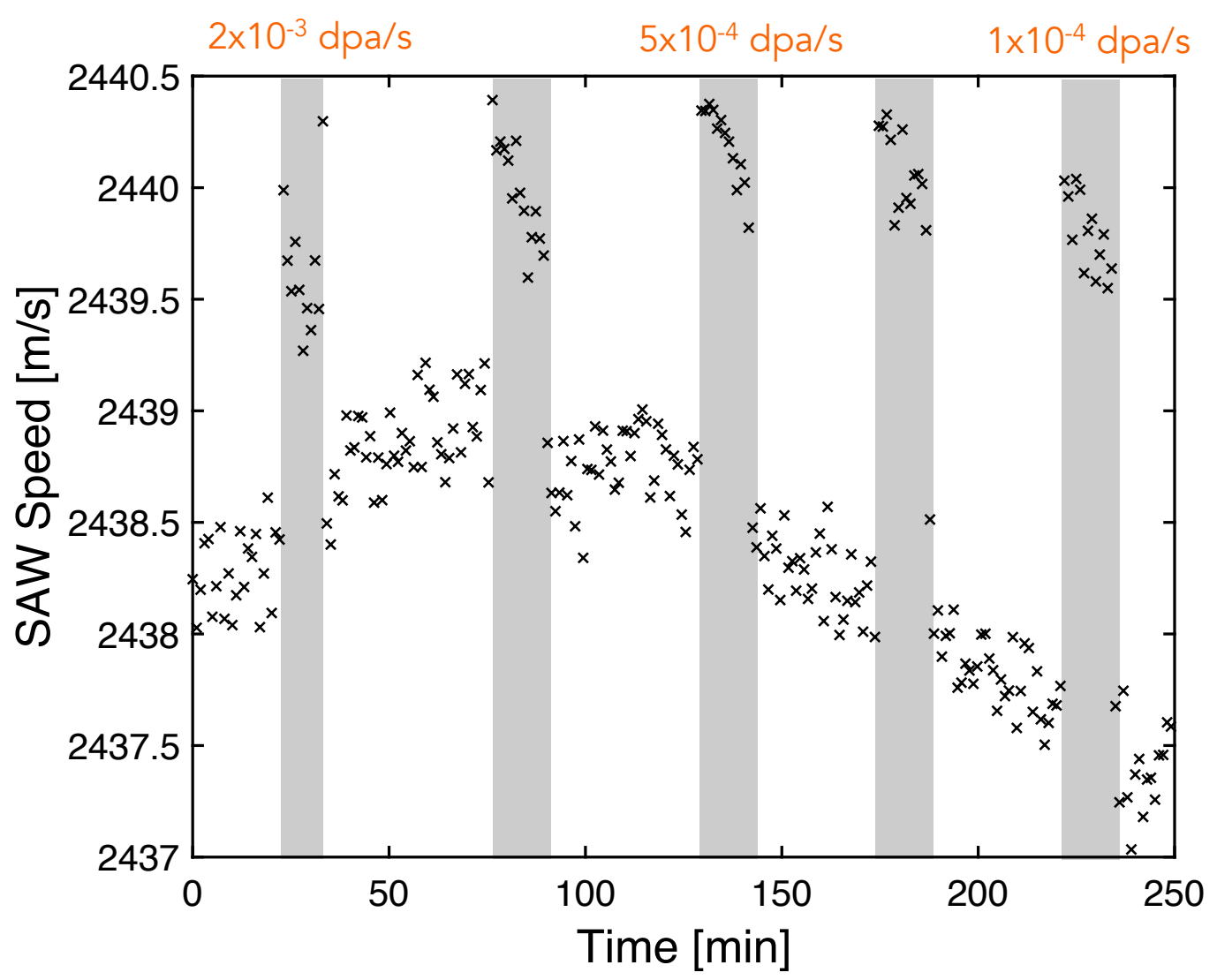

transient defect populations stiffen the elastic modulus, inversely correlated with dose rate

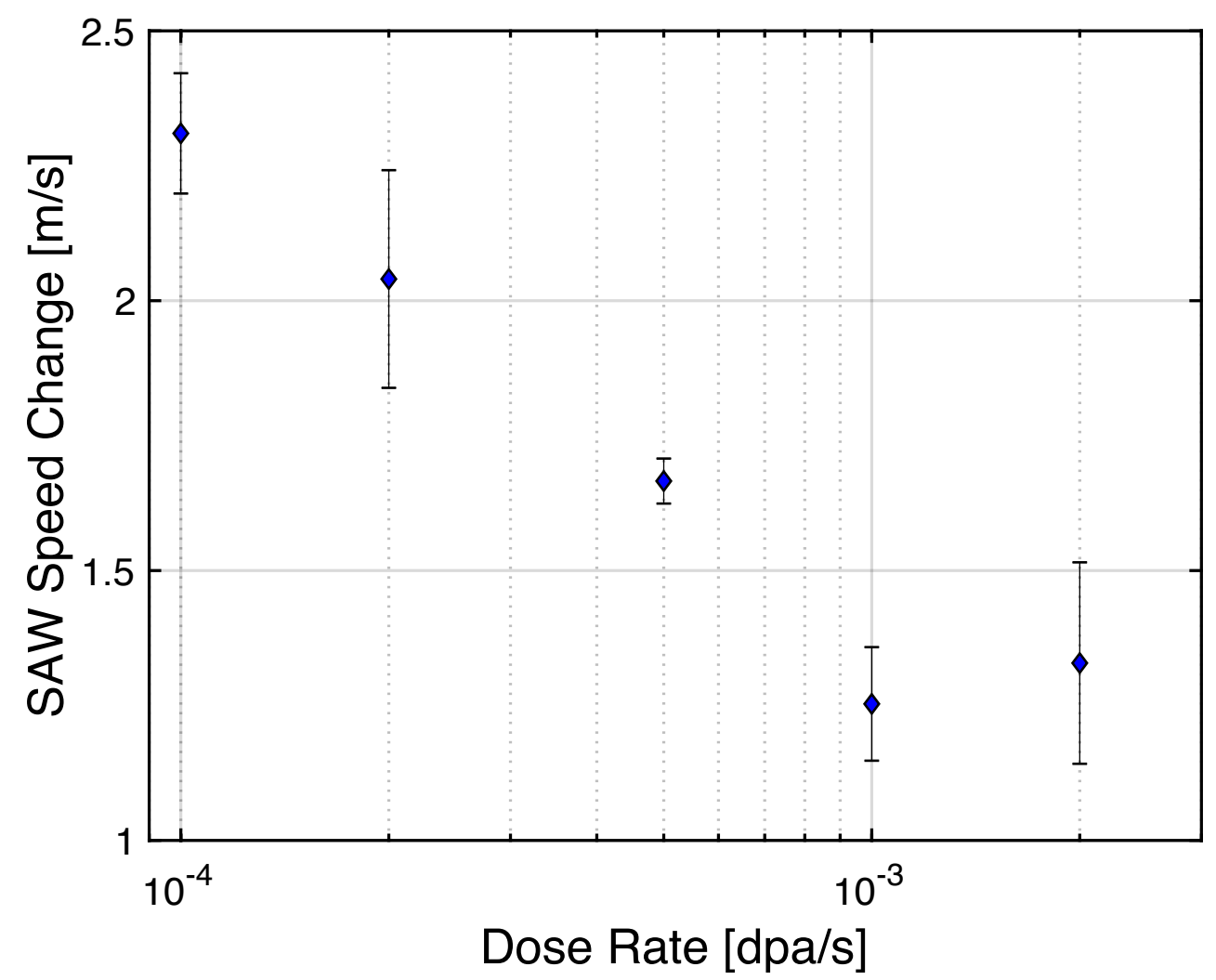

background defect accumulation continually evolving and not fully annealing 


\section{$\mathrm{NiFe}$}
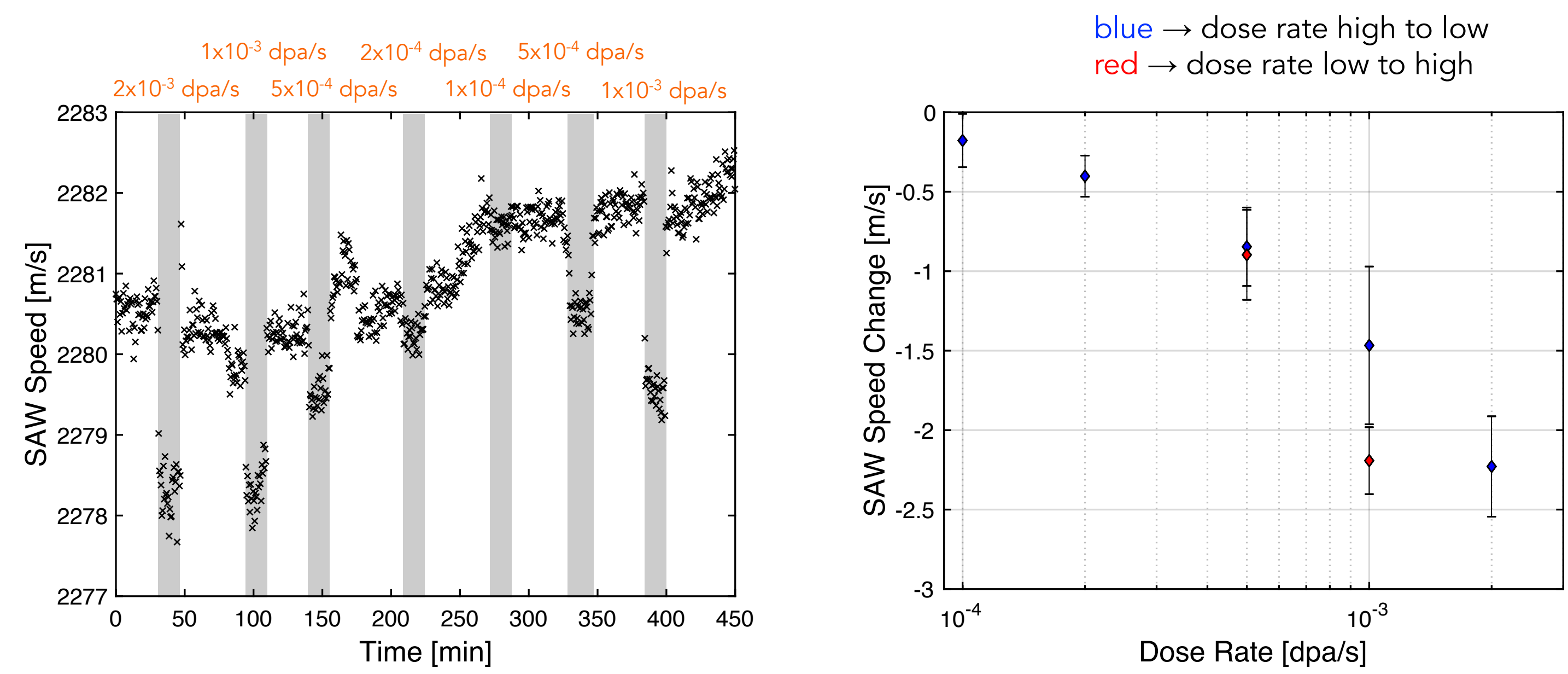

transient defect populations soften/reduce the elastic modulus, magnitude directly correlated with dose rate 


\section{$\mathrm{NiCoCr}$}

$1 \times 10^{-3} \mathrm{dpa} / \mathrm{s} \quad 2 \times 10^{-4} \mathrm{dpa} / \mathrm{s}$

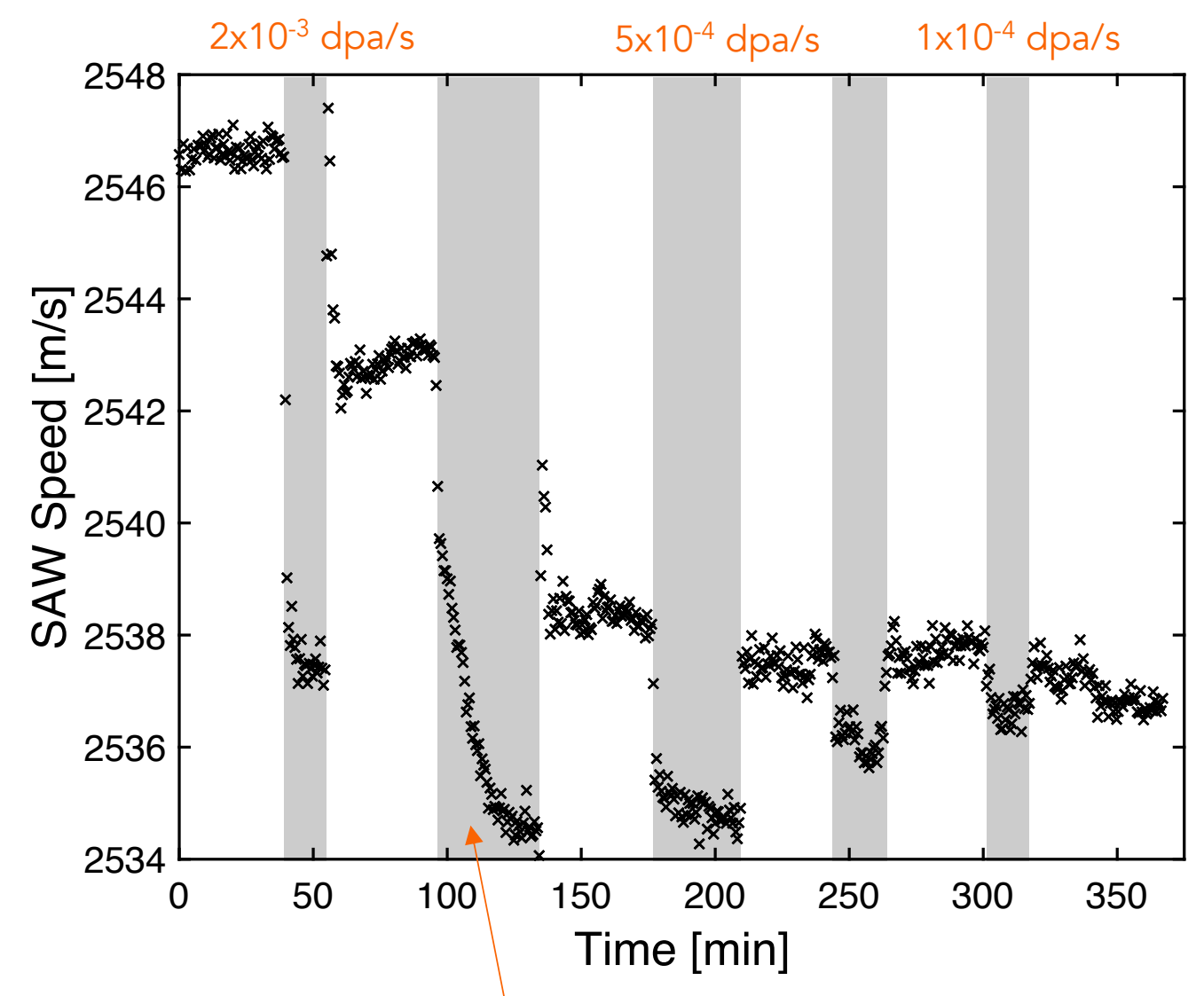

elastic constant reduction greatest observed of any alloy chemistry studied

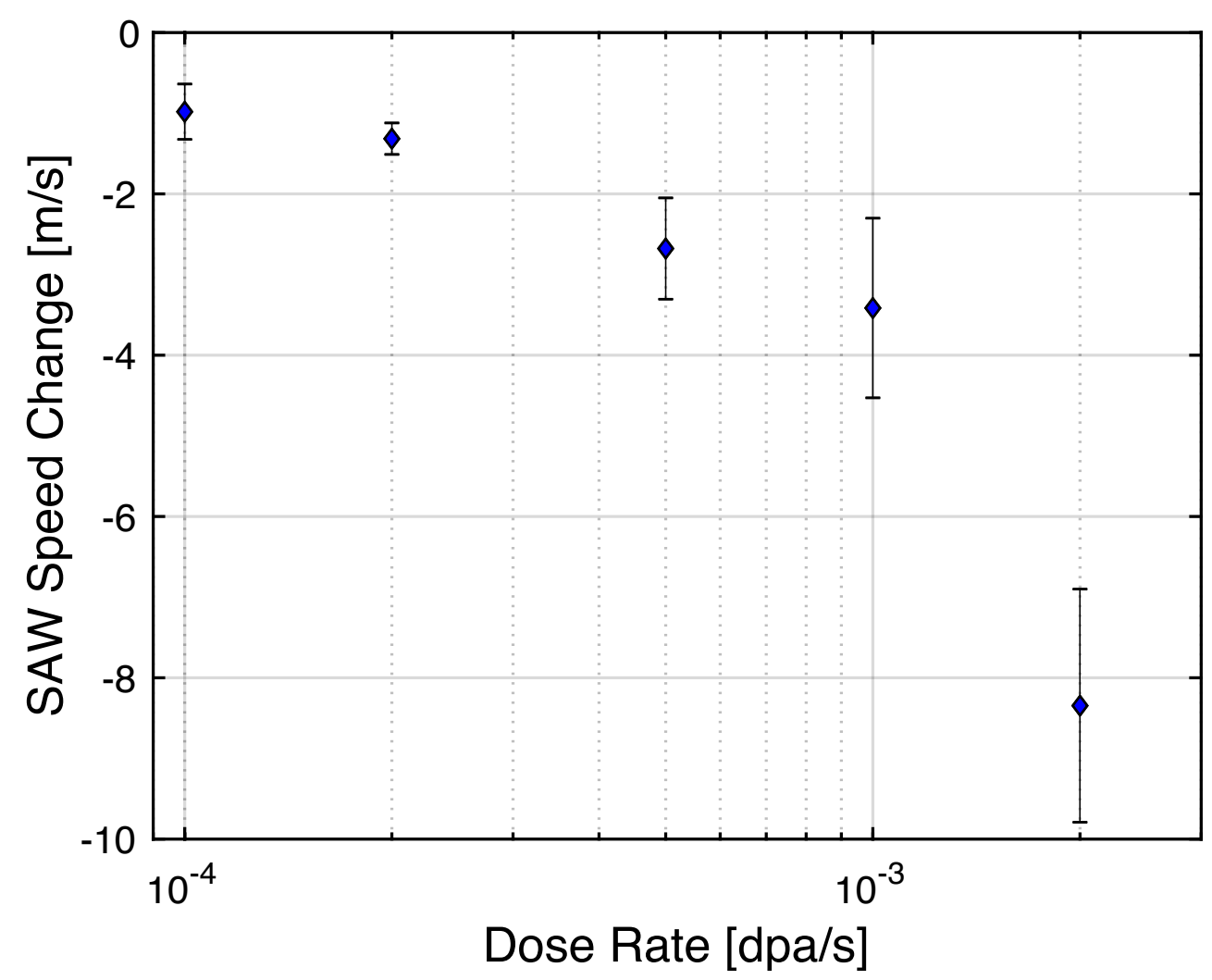

transient accumulation time longer than for other alloys 


\section{$\mathrm{NiFeCoCr}$}

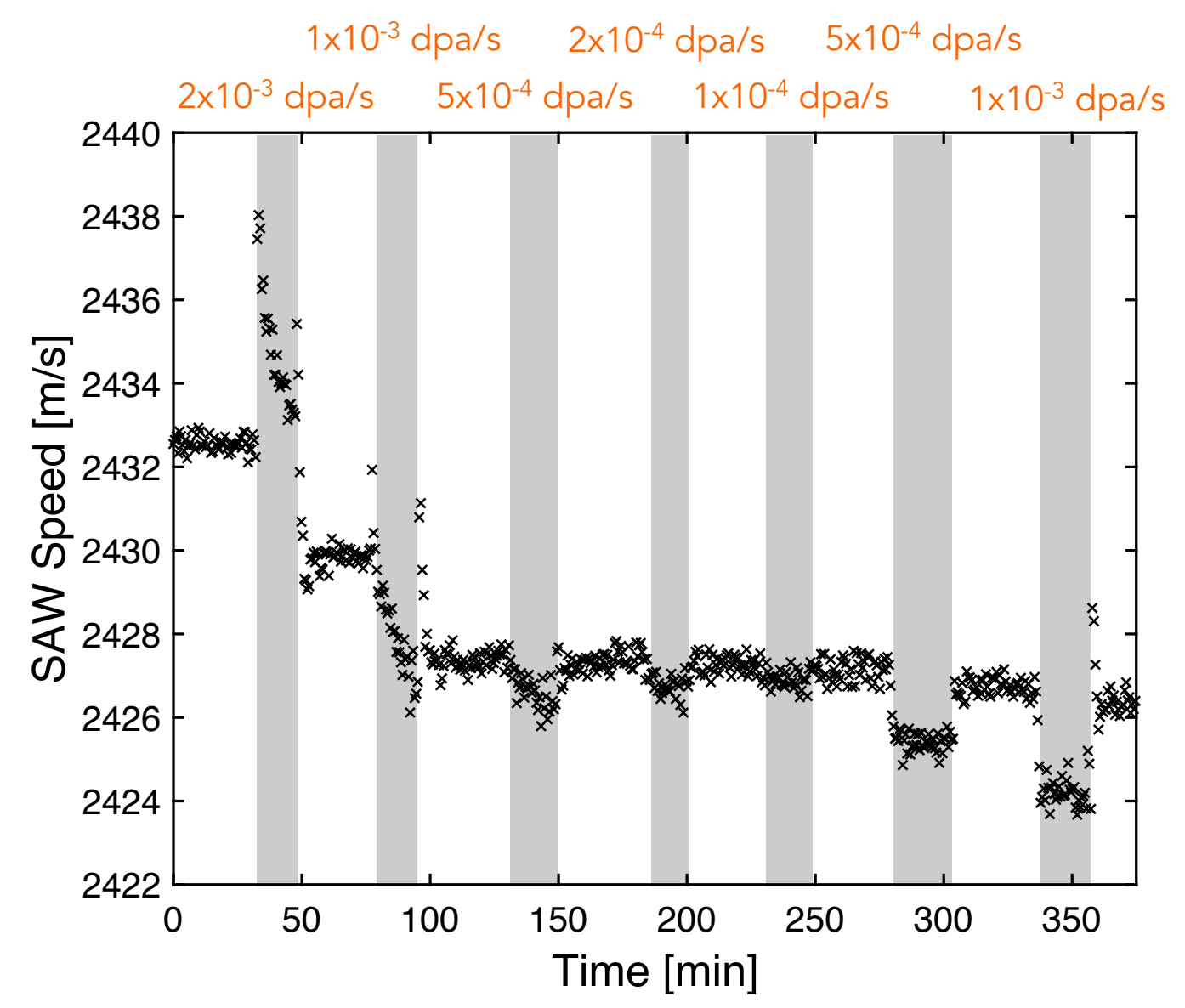

blue $\rightarrow$ dose rate high to low red $\rightarrow$ dose rate low to high

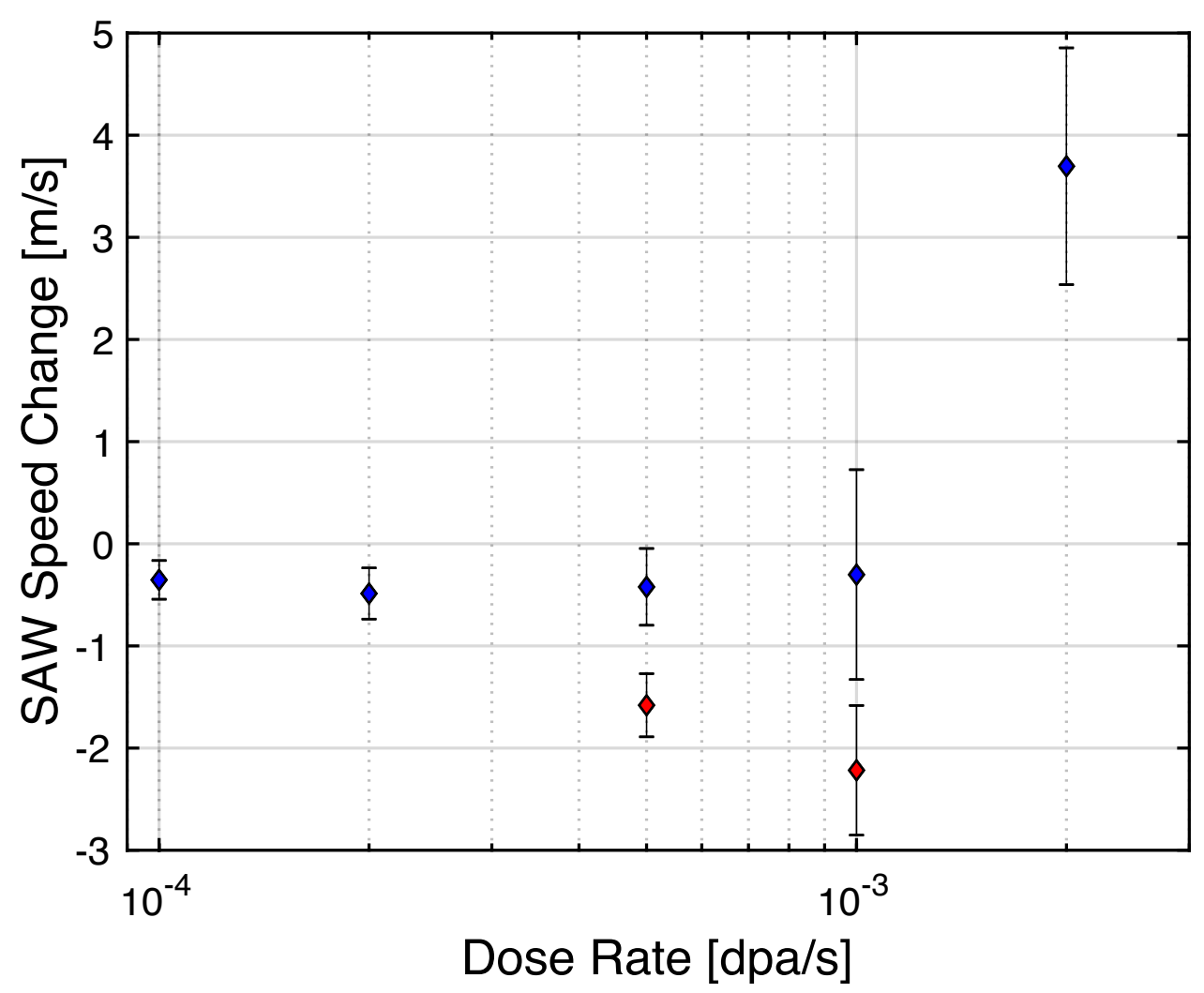

initial lattice stiffening is irreversible, softening observed at identical dose rates following saturation 


\section{short-timescale defect accumulation observations and question}

stiffening vs. softening in different alloys defect type variation?

limitations of subsequent irradiation impulses underlying background evolution? higher temperatures needed for annealing? 


\section{in situ means} interesting failure

13Cr-7Al-23Zr-30Mo-24Nb-4Ta

refractory multiple principle element alloy

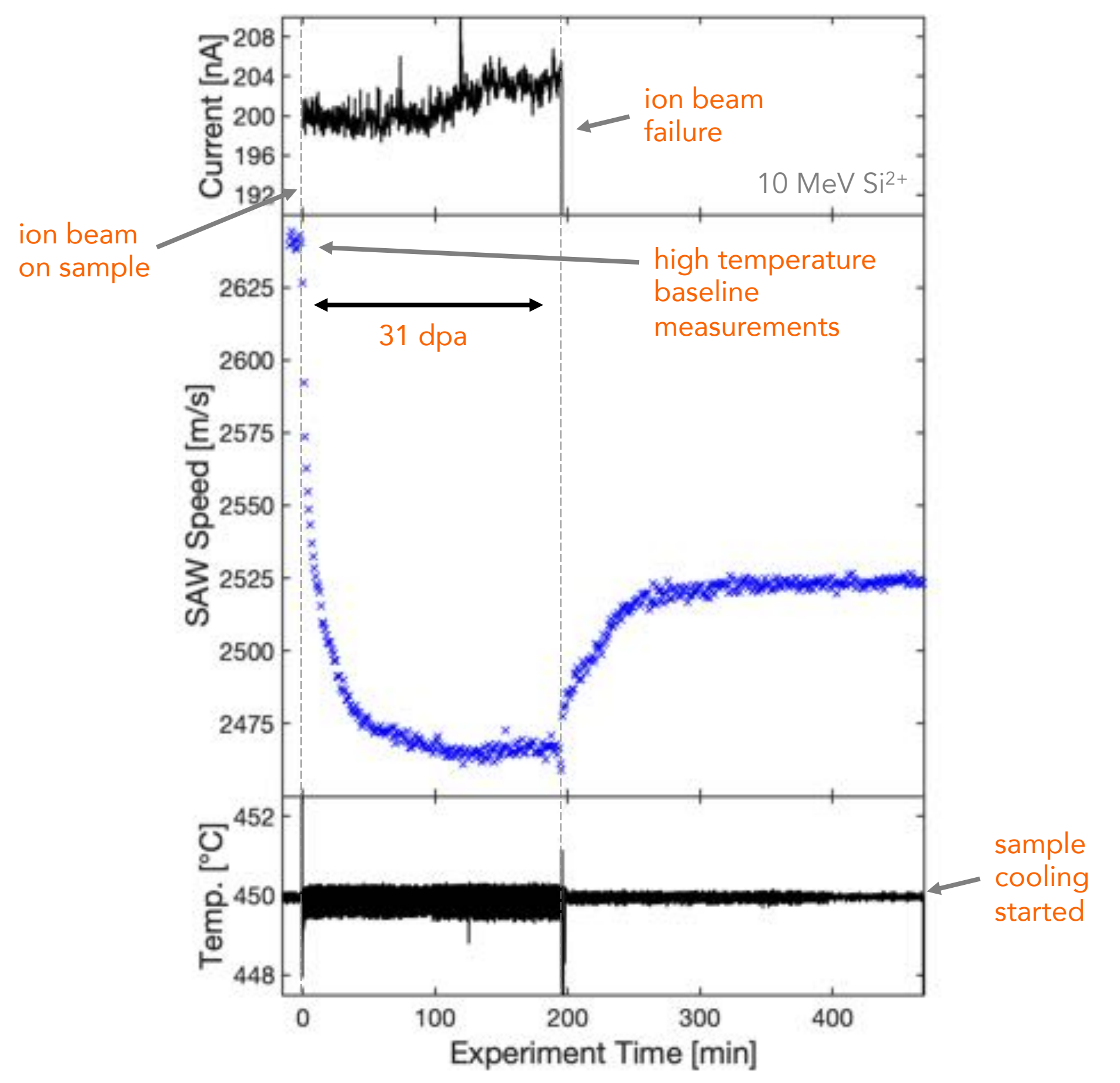


- in situ ion irradiation TGS beamline available for use at Sandia National Labs

- thermoelastic material properties explored directly in situ under extreme conditions at both short and long timescales
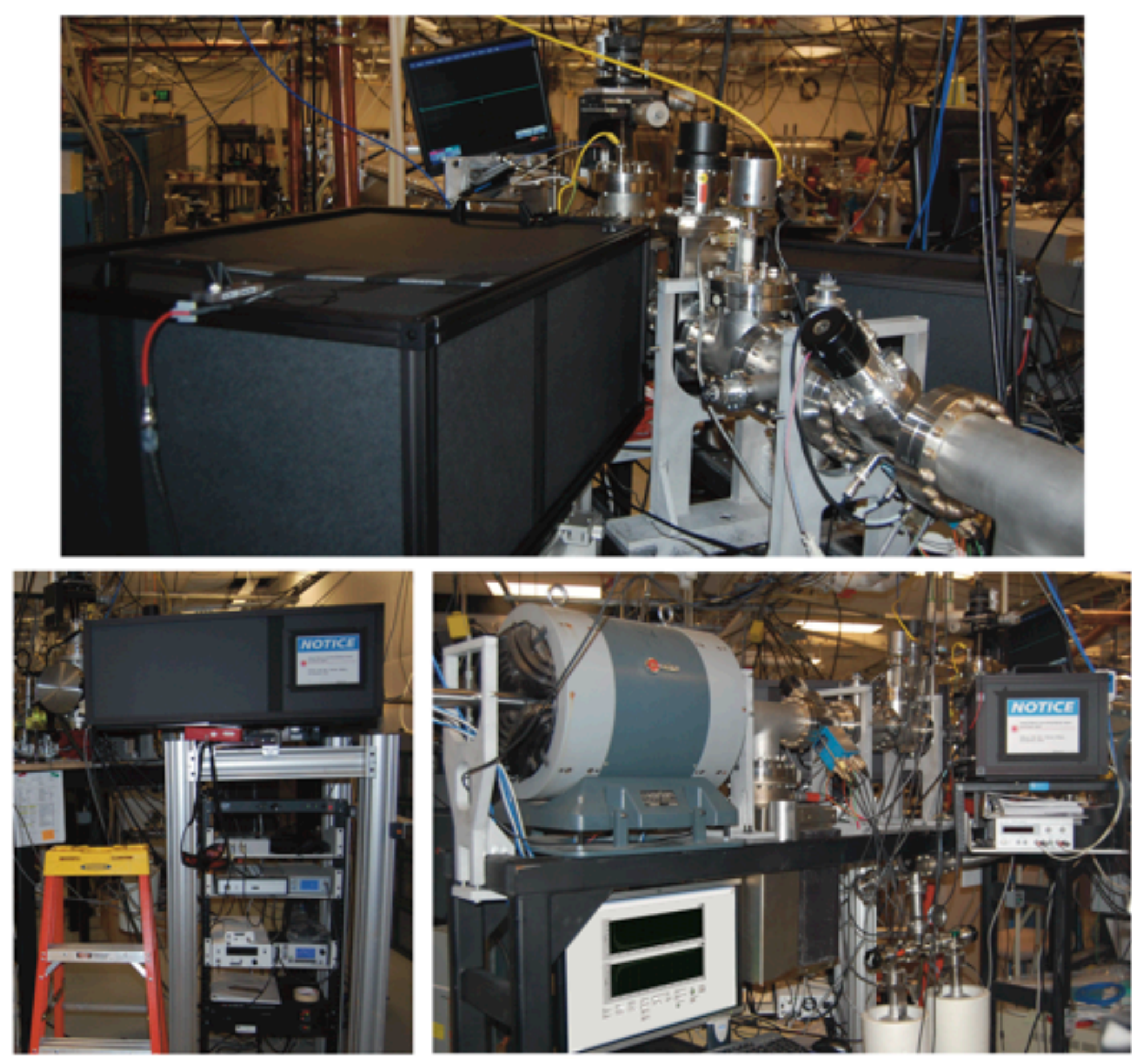


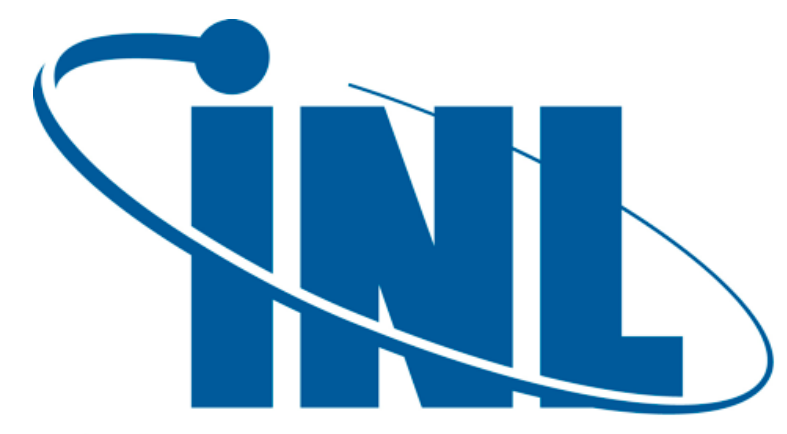

Idaho National Laboratory

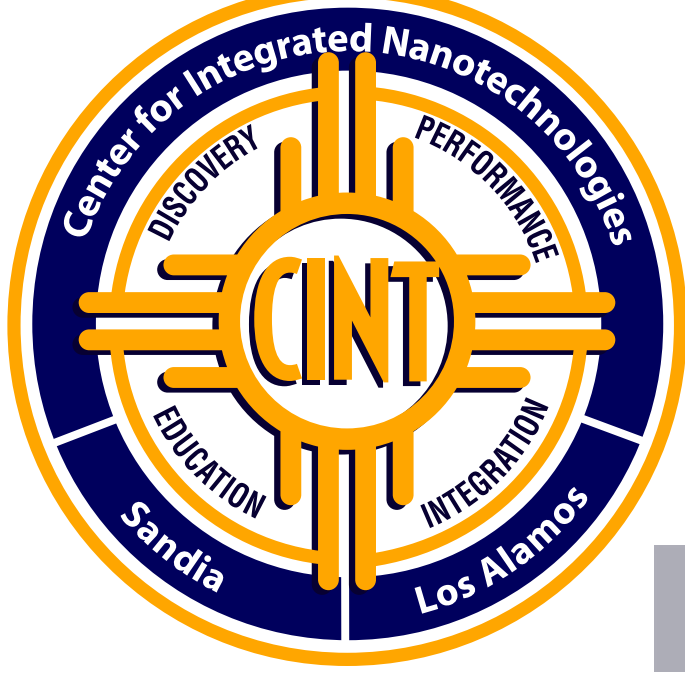

This work was performed, in part, at the Center for Integrated Nanotechnologies, an Office of Science User Facility operated for the U.S. Department of Energy (DOE) Office of Science. Sandia National Laboratories is a multi-mission laboratory managed and operated by National Technology and Engineering Solutions of

Sandia, LLC., a wholly owned subsidiary of Honeywell International, Inc., for the U.S. DOE's National Nuclear Security Administration under contract DE-NA-0003525. The views expressed in the presentation do not

necessarily represent the views of the U.S. DOE or the United States Government. 
- in situ ion irradiation TGS beamline available for use at Sandia National Labs

- thermoelastic material properties explored directly in situ under extreme conditions at both short and long timescales

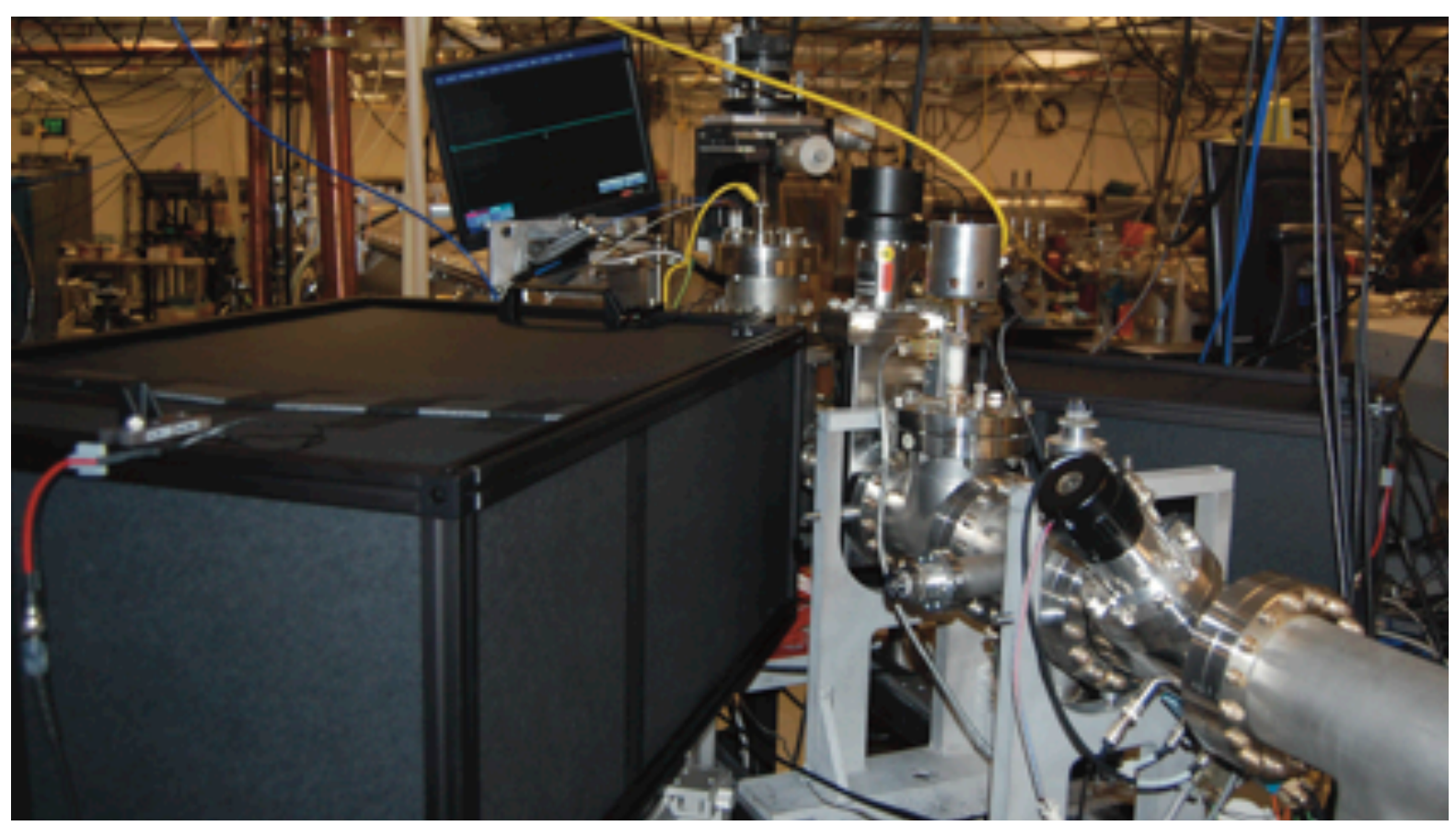

contact: cody.dennett@inl.gov

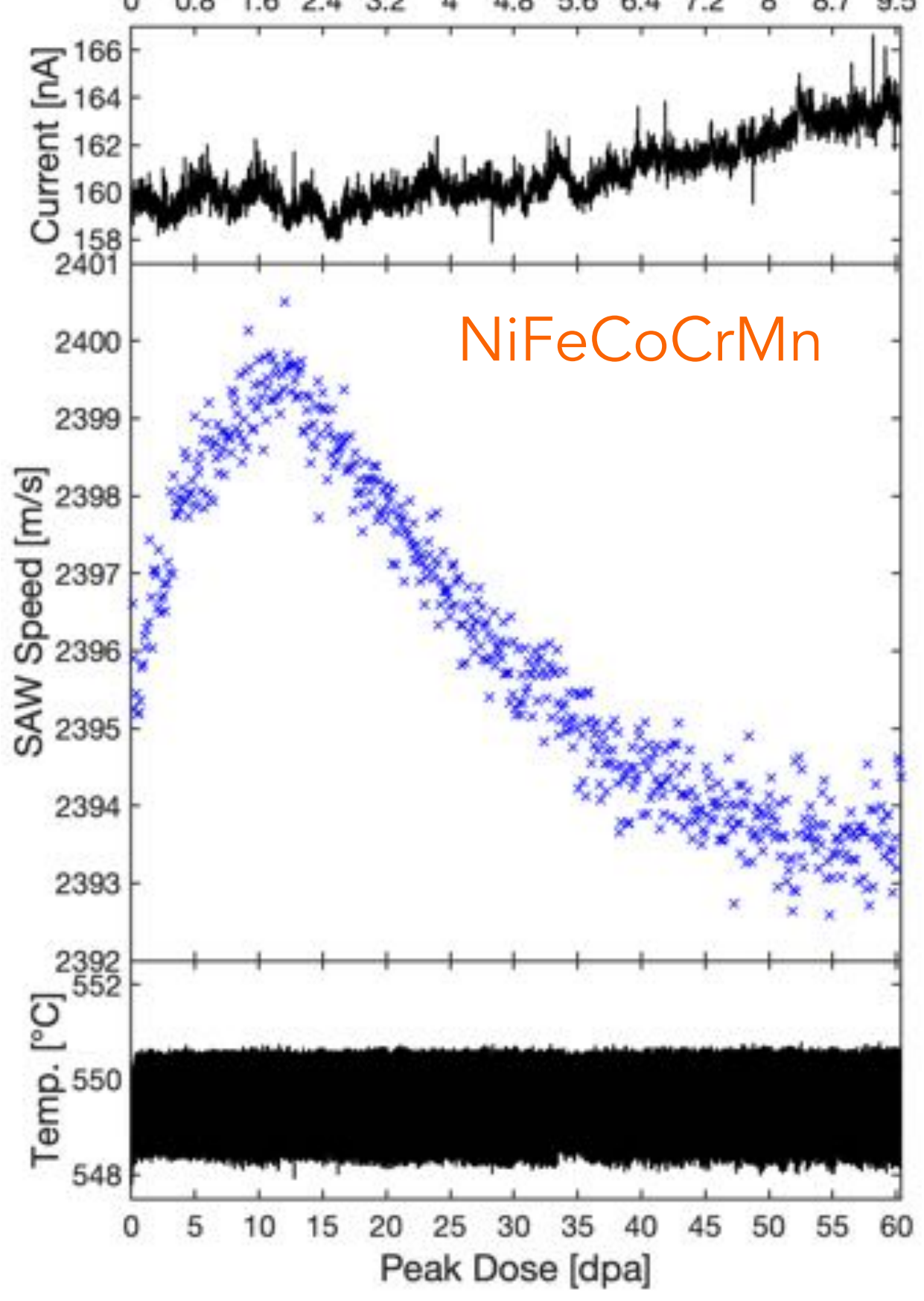




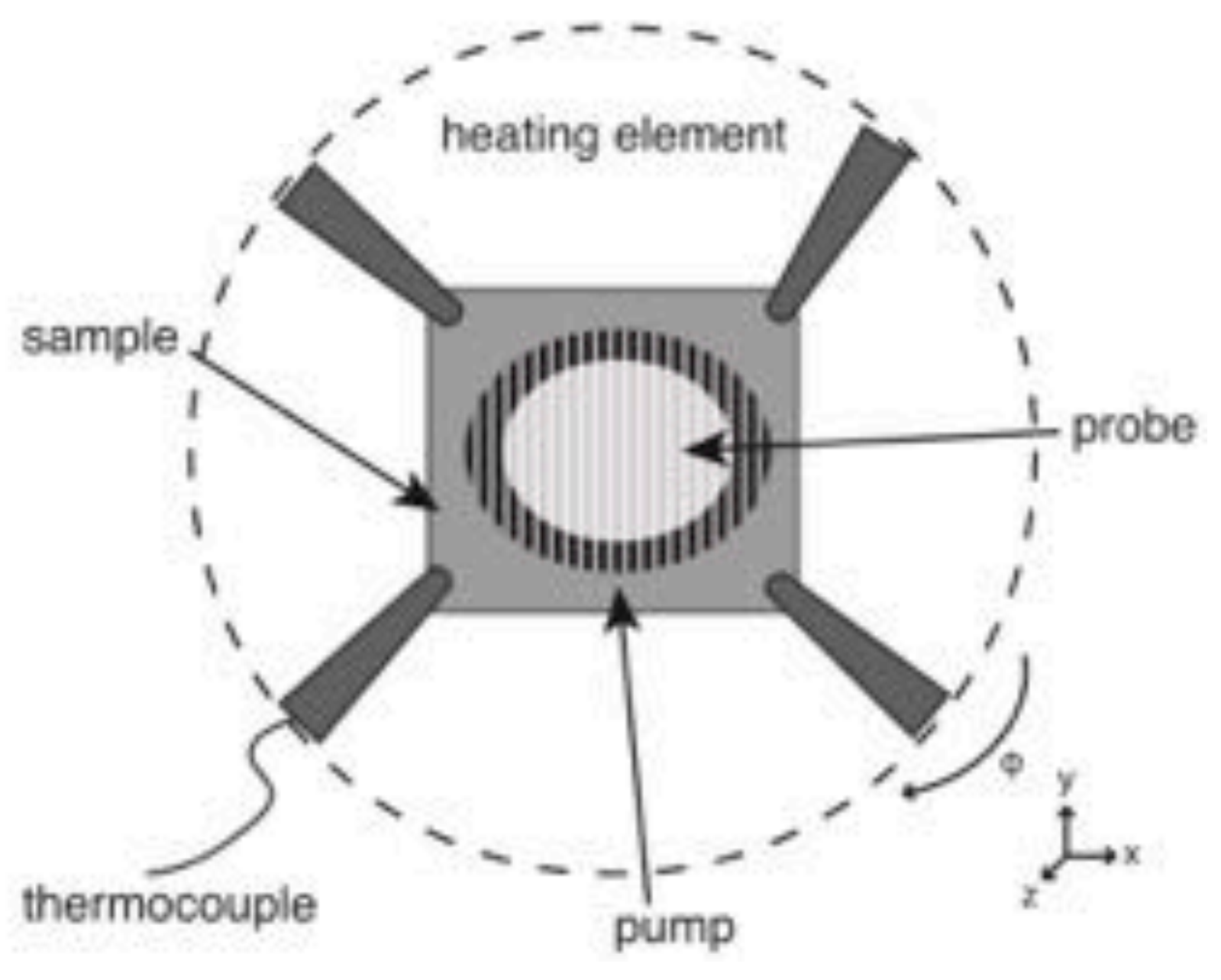

sample in place

high temp. operation

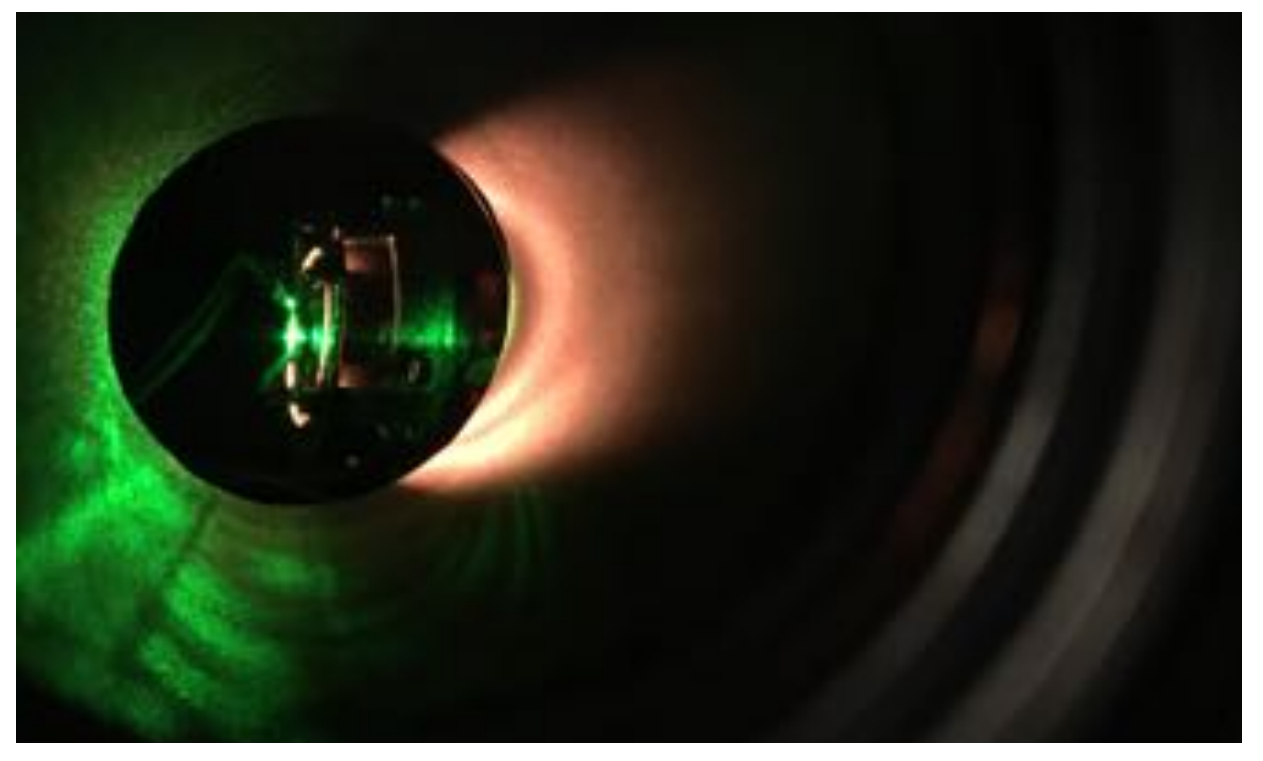

